\title{
The effects of high versus low talker variability and individual aptitude on phonetic training of Mandarin lexical tones
}

\author{
Hanyu Dong ${ }^{\text {Corresp., } 1}$, Meghan Clayards $^{2}$, Helen Brown $^{3}$, Elizabeth Wonnacott $^{\text {Corresp. } 1}$ \\ 1 Division of Psychology and Language Sciences, University College London, London, United Kingdom \\ 2 Department of Linguistics, School of Communications Sciences and Disorders, McGill University, Montreal, QC, Canada \\ 3 Department of Psychology, Nottingham Trent University, Nottingham, United Kingdom \\ Corresponding Authors: Hanyu Dong, Elizabeth Wonnacott \\ Email address: hanyu.dong.10@ucl.ac.uk, e.wonnacott@ucl.ac.uk
}

High variability training has been found to be more effective than low variability training when learning various non-native phonetic contrasts. However, little research has considered whether this applies to the learning of tone contrasts. The only two relevant studies suggested that the effect of high variability training depends on the perceptual aptitude of participants (Perrachione, Lee, Ha, \& Wong, 2011; Sadakata \& McQueen, 2014). The present study extends these findings by examining the interaction between individual aptitude and input variability using natural, meaningful second language input (both previous studies used pseudowords). Sixty English speakers took part in an eight session phonetic training paradigm. They were assigned to high/low/high-blocked variability training groups and learned real Mandarin tones and words. Individual aptitude was measured following previous work. Learning was measured using one discrimination task, one identification task and two production tasks. All tasks assessed generalisation. All groups improved in both the production and perception of tones which transferred to untrained voices and items, demonstrating the effectiveness of training despite the increased complexity compared with previous research. Although the low variability group exhibited an advantage with the training stimuli, there was no evidence for a benefit of high-variability in any of the tests of generalisation. Moreover, although aptitude significantly predicted performance in discrimination, identification and training tasks, no interaction between individual aptitude and variability was revealed. Additional Bayes Factor analyses indicated substantial evidence for the null for the hypotheses of a benefit of high-variability in generalisation, however the evidence regarding the interaction was ambiguous. We discuss these results in light of previous findings. 

The effects of high versus low talker variability and individual aptitude on phonetic training of Mandarin lexical tones Hanyu Dong ${ }^{1}$, Meghan Clayards ${ }^{2}$, Helen Brown $^{3}$, \& Elizabeth Wonnacott ${ }^{1}$ ${ }^{1}$ Division of Psychology and Language Sciences, University College London, London, UK University, Montreal, QC, Canada

11 Correspondence concerning this article should be addressed to Elizabeth Wonnacott, Division of

12 Psychology and Language Sciences, Chandler House, 2 Wakefield Street, London, WC1N 1PF.

13 Email: e.wonnacott@ucl.ac.uk 
21 We would like to thank Catriona Silvey for her help with the Bayesian analyses. Also Zoltan Dienes for his general advice concerning best practice in computing Bayes Factors. 


\section{Abstract}

25 High variability training has been found to be more effective than low variability training when

26 learning various non-native phonetic contrasts. However, little research has considered whether

27 this applies to the learning of tone contrasts. The only two relevant studies suggested that the

28 effect of high variability training depends on the perceptual aptitude of participants (Perrachione,

29 Lee, Ha, \& Wong, 2011; Sadakata \& McQueen, 2014). The present study extends these findings

30 by examining the interaction between individual aptitude and input variability using natural,

31 meaningful second language input (both previous studies used pseudowords). Sixty English

32 speakers took part in an eight session phonetic training paradigm. They were assigned to

33 high/low/high-blocked variability training groups and learned real Mandarin tones and words.

34 Individual aptitude was measured following previous work. Learning was measured using one

35 discrimination task, one identification task and two production tasks. All tasks assessed

36 generalisation. All groups improved in both the production and perception of tones which

37 transferred to untrained voices and items, demonstrating the effectiveness of training despite the

38 increased complexity compared with previous research. Although the low variability group

39 exhibited an advantage with the training stimuli, there was no evidence for a benefit of high-

40 variability in any of the tests of generalisation. Moreover, although aptitude significantly

41 predicted performance in discrimination, identification and training tasks, no interaction between

42 individual aptitude and variability was revealed. Additional Bayes Factor analyses indicated

43 substantial evidence for the null for the hypotheses of a benefit of high-variability in

44 generalisation, however the evidence regarding the interaction was ambiguous. We

45 discuss these results in light of previous findings. 


\section{Introduction}

One challenging aspect of learning a second language (L2) is learning to accurately

48

49

perceive non-native phonetic categories. This task is particularly difficult when the L2 relies on the same acoustic dimensions as the first language (L1), but for different purposes (Bygate, Swain, \& Skehan, 2013), suggesting that it is challenging to adjust existing acoustic properties in the L1 to learn new L2 categories. This challenge is compounded by the fact that speech is highly variable in the natural linguistic environment. Variability comes not only from the phonetic context but also from differences between speakers. Thus, learners must learn to distinguish the new L2 categories despite all the variability present in the learning input. There is evidence that native listeners can process this variability in speech faster and more accurately than non-native listeners (Bradlow \& Pisoni, 1999), indicating that variability is indeed a challenge for L2 learners. Despite this, it has been suggested that input variability may be beneficial for L2 learning and generalisation (Barcroft \& Sommers, 2005; Lively, Logan \& Pisoni, 1993). However recent evidence suggests that the ability to benefit from variability may depend on individual learner aptitude (Perrachione et al., 2011; Sadakata \& McQueen, 2014), at least in the learning of lexical tones (i.e. the distinctive pitch patterns carried by the syllable of a word which, in certain languages, distinguish meaningful lexical contrasts). The current paper further explores how and when variability supports or impedes learning of new L2 phonetic categories, focusing on English learners of Mandarin tone contrasts.

\subsection{High Variability L2 Phonetic Training for Non-Tonal Contrasts}

A substantial body of literature has explored whether phonetic training can be used to improve identification and discrimination of non-native phonetic contrasts in L2 learners. An early study by Strange and Dittman (1984) attempted to train Japanese speakers on the English 
$69 / / \mathrm{r} / \mathrm{-} / \mathrm{l} /$ distinction, a phoneme contrast that does not exist in Japanese. Participants were trained

70 on stimuli from a synthetic rock-lock continuum. The key result was that although performance

71 increased both for trained and novel synthetic items, participants failed to show any

72 improvement for naturally produced minimal pair items. Later research suggested that a key

73 factor which prevented generalisation to natural speech tokens was a lack of variability in the

74 training materials: Variability was present in the form of the ambiguous intermediate stimuli

75 along the continuum, however, there was a single phonetic context and a single (synthesised)

76 speaker. Logan, Lively, and Pisoni (1991) also trained Japanese learners on the English /r/-/1/

77 contrast, but included multiple natural exemplars spoken by six speakers, with the target speech

78 sounds appearing in a range of phonetic contexts. In contrast to Strange and Dittman, they found

79 that participants successfully generalised both to new speakers and new words at test. This was

80 the first study to indicate the importance of variability within the training materials. A follow up

81 study by Lively et al. (1993) provided further evidence for this by contrasting a condition with

82 high variability input to one with low variability input in which the stimuli were spoken by a

83 single speaker (although still exemplified in multiple phonetic contexts). Participants in the low

84 variability group improved during the training sessions but failed to generalise this learning to a

85 new speaker.

$86 \quad$ Following Lively et al. (1993) high variability phonetic training (HVPT) has become

87 standard in L2 phonetic training. This methodology has been successfully extended to training a

88 variety of contrasts in various languages such as learning of the English /u/-/ / distinction by

89 Catalan/Spanish bilinguals (Aliaga-García \& Mora, 2009), learning of the English /i/-/I/ contrast

90 by native Greek speakers (Giannakopoulou, Uther \& Ylinen, 2013; Lengeris \& Hazan, 2010), 
91 and learning of the English /w/-/v/ distinction by native German speakers (Iverson, Ekanayake,

92 Hamann, Sennema, \& Evans, 2008).

93 There is also some evidence that this type of perceptual training benefits production in

94 addition to perception. Bradlow, Akahane-Yamada, Pisoni and Tohkura (1999) found that

95 production of the $/ \mathrm{r} /-/ 1 /$ contrast improved in Japanese speakers following HVPT, with this

96 improvement being retained even after three months. Similar improvement on the production of

97 American English mid to low vowels by Japanese speakers following HVPT was also reported

98 by Lambacher, Martens, Kakehi, Marasinghe, and Molholt (2005). However, the evidence here

99 is mixed: A recent study by Alshangiti and Evans (2014) employed HVPT to train Arabic

100 learners on non-native English vowel contrasts and found no improvements in production,

101 although participants receiving additional explicit production training did show some limited

102 improvement.

103 Although the studies reviewed above all used HVPT, only the original work by Logan

104 and colleagues directly contrasted the use of high and low variability materials. It is notable these

105 seminal experiments used small samples (the tests of generalisation were administered to only

106 three of the participants in Logan et al., 1991). Since then, few studies have explicitly contrasted

107 high and low variability training. One such study was Sadakata and McQueen (2013), who

108 trained native Dutch speakers with geminate and singleton variants of the Japanese fricative /s/.

109 Participants were trained with either a limited set of words recorded by a single speaker (low

110 variability) or with a more variable set of words recorded by multiple speakers (high variability).

111 Both types of training led to increases in generalisation to untrained fricatives and speakers.

112 However, in an identification task, the improvement was greater for participants receiving high

113 variability training than those receiving low variability training. Similar results were reported by 
114 Wong (2014) who trained native Cantonese speakers with the English /e/ - /æ/ contrast. Both low

115 variability (1 speaker) and high variability (6 speakers) training lead to increased performance

116 from pre- to post- test, but the improvement was greater for the high variability group. This was

117 found in tests of generalisation to new speakers and new items, and from perception to

118 production. In contrast, a recent phonetic training study did not find the same benefit.

119 Giannakopoulou, Brown, Clayards, and Wonnacott (2017) compared matched high variability

120 (four speakers) and low variability (one speaker) training for adult and child (8-year-old) native

121 Greek speakers who were trained on the English /i/-/I/ contrast. This study did not show a benefit

122 for high variability compared to low variability training in either age group, even for

123 generalisation items. However, for adult participants, it is unclear the extent to which this was

124 due to ceiling effects. To our knowledge, the only other previous studies that specifically

125 manipulated variability during learning of non-native phonetic categories are those by

126 Perrachione et al. (2011), and Sadakata and McQueen (2014), which both looked at the learning

127 of lexical tone. We discuss these studies in more detail in the following section.

128 Although there is a relatively small evidence base regarding a benefit of high over low

129 phonetic training for non-native phoneme categories, there is further evidence for this benefit in

130 related areas of speech and language learning, specifically accent categorisation and adaptation

131 (Bradlow \& Bent, 2008; Clopper \& Pisoni, 2004), and L2 vocabulary learning (Barcroft \&

132 Sommers, 2005, 2014; Sommers \& Barcroft, 2007, 2011). Benefits of HVPT are generally seen

133 in tasks of generalisation, suggesting that exposure to variation across speakers and/or items

134 boosts the ability to generalise across these dimensions. This intuitively sensible result is in line

135 with the predictions of computational models in which irrelevant contextual/speaker identity

136 cues compete with phonetically relevant cues, so that dissociation of these irrelevant cues is the 
137 key mechanism which underpins generalisation (Apfelbaum \& McMurray, 2011; Ramscar \&

138 Baayen, 2013; Ramscar, Yarlett, Dye, Denny \& Thorpe, 2010).

$139 \quad 1.2 \quad$ Phonetic Training of L2 Lexical Tones

140 Each of the phonetic training studies discussed above involved training a segmental

141 contrast (consonantal or vocalic). Lexical tone is another type of phonological contrast in some

142 natural languages, whereby the pitch contour is used to distinguish lexical or grammatical

143 meanings (Yip, 2002). For example, Mandarin Chinese has four lexical tones: level-tone (Tone

144 1), rising-tone (Tone 2), dipping-tone (Tone 3) and falling-tone (Tone 4). These pitch contours

145 combine with syllables to distinguish meanings. For instance, the syllable $b a$ combines with the

146 four tones to mean: eight (bāa, Tone 1), pluck (bá, Tone 2), grasp (bă, Tone 3) and father (bà,

147 Tone 4). Each of these words thus forms a minimal pair with each of the others. Note that while

148 non-tonal languages such as English use pitch information extensively for intonation (e.g.

149 forming a question, or for emphasis), and that pitch plays a role in marking stress at the lexical

150 level in (e.g. 'import/im'port), this is quite different from a lexical tone system, causing

151 difficulties for L2 learners of Mandarin.

152 The first study examining lexical tone training was conducted by Wang, Spence,

153 Jongman, and Sereno (1999). A similar paradigm to that used by Logan et al. (1991) was

154 adopted using four speakers for training. Training materials were all real monosyllabic Mandarin

155 words that varied in the consonants, vowels and syllable structure. During training participants

156 heard a syllable whilst viewing two of the four standard diacritic representations (i.e., $\rightarrow, \nearrow, \vee, 〉$,

157 which are iconic in nature). They were asked to pick out the picture of the arrow that

158 corresponded to the tone. At test, participants chose which tone they had heard out of a choice of

159 all four diacritics. There were also two generalisation tasks, one testing generalisation to 
160 untrained items and one testing generalisation to a new speaker. Native American English

161 speakers showed significant improvement in the accuracy of tone identification after eight

162 sessions of high variability training over two weeks, and this generalised to both new words and

163 a new speaker. In a follow up study, Wang, Jongman and Sereno (2003) used the same training

164 paradigm to test whether learning transferred to production. They recruited participants taking

165 Mandarin courses and asked them to read through a list of 80 Mandarin words written in Pinyin

166 (an alphabetic transcription) before and after training. They found improvements in production,

167 although these were mainly seen in pitch contour rather than pitch height.

168 These studies suggested that as with segmental phoneme contrasts, high variability

169 training may also facilitate the learning of tone contrasts. However, Wang and colleagues (1999,

170 2003) did not directly contrast high and low variability training materials. Perrachione et al.

171 (2011) investigated this contrast directly. They trained native American English speakers with no

172 previous knowledge of Mandarin (or any other tonal language), using English monosyllabic

173 pseudowords combined with Mandarin tones 12 , and $4(\rightarrow, \nearrow \& \searrow)$. The training task used

174 either low variability (one speaker) or high variability (four speaker) input. During the training,

175 participants matched the sound they heard with one of three pictures of concrete objects

176 presented, where the three words associated with these pictures were minimal trios that differed

177 only in tone. Participants were tested on their ability to generalise their learning to new speakers.

178 Importantly, Perrachione et al. (2011) were also interested in the role of individual differences in

179 learning. Therefore, they also determined participants' baseline ability to perceive the tone

180 contrasts prior to training using a Pitch Contour Perception Test. In this task, participants heard a

181 vowel produced with either Mandarin tone 1, 2 or 4 whilst viewing pictures of standard diacritics

182 associated with these tones $(\rightarrow, \nearrow \& \searrow)$, and were asked to select the arrow that corresponded to 
183 the tone. Based on performance in this task, the researchers grouped participants into high and

184 low aptitude groups. The results showed that whilst the low variability group outperformed the

185 high variability group during training (presumably due to accommodation to a repeated speaker

186 throughout the task), there were no differences between the high and low variability groups

187 during test. Critically however, there was an interaction between an individuals' aptitude

188 categorisation and the type of variability training: Only participants with high aptitude benefitted

189 from high variability training, while those with low aptitude actually benefitted more from low

190 variability training. It is important to note that this interaction was seen in a task which relied on

191 participants' ability to generalise their learning ${ }^{1}$ of tones to an untrained speaker. That is, in a

192 task where we would expect that exposure to multiple speakers would be beneficial since it

193 should allow learners to better dissociate the tones from the particular speakers used in training.

194 These results, therefore, suggest that only the high aptitude learners can take advantage of this

195 benefit. Another training study by Sadakata and McQueen (2014) also explored the relationship

196 between input variability and individual aptitude in lexical tone training, though using different

197 training and testing materials. They trained native Dutch speakers (with no prior knowledge of

198 Mandarin or any other tonal language) using naturally produced bisyllabic Mandarin

199 pseudowords. The two syllables in each word either had Tone 2 followed by Tone 1, or Tone 3

200 followed by Tone 1, and each tone pair was randomly assigned one of two numeric labels (e.g.

201 for one participant Tone 2-Tone 1 was labelled "1", Tone 3-Tone 1 was labelled "2"). During the

202 training task, participants identified the tone pair type of each stimulus by choosing the correct

\footnotetext{
${ }^{1}$ In their paper, Perrachione et al (2011) do not refer to this task as a generalisation task. Instead they report a generalisation measure which is a ratio of performance on this test with novel speakers to performance in training (test-performance/training-performance). Note that this ratio will increase not only if participants are better at test, but also if they are worse in training. Using this measure, Perrachione et al. found a benefit of high variability training. However on inspection of the means, it seems that this relationship is driven by the poorer performance in training in the high variability condition, rather than by better performance in the test with novel speakers. We therefore do not see the ratio measure as providing evidence for an overall benefit of $\mathrm{HV}$ training on generalisation.
} 
203 numeric label (e.g. hear /pasa/ with Tone 2-Tone 1, correct response is 1). Thus, in contrast to the 204 study by Perrachione et al. (2011), participants did not need to learn the meaning of each word.

205 Input variability was manipulated, with three levels (low/medium/high). In contrast to the work 206 by Perrachione et al., where the high variability and low variability conditions differed only in 207 terms of the number of speakers, in this study variability was increased both by including more 208 speakers and more items. Specifically, the number of different vowels used in the bi-syllabic 209 sequences was manipulated: the low variability group encountered only one vowel (.e.g. pasa, 210 casa, lasa, etc.) whereas the medium and high variability groups encountered four different

211 vowels (pasa, pesa, pisa, pusa; casa, cesa, cisa, cusa; lasa, lesa, lisa, lusa etc.). Participants were

212 tested on the trained items (i.e. using trained speakers and trained items). Generalisation was also

213 examined in a number of ways by looking at (1) trained items spoken by an untrained talker; (2)

214 pseudowords containing untrained vowels (3) pseudowords in which the order of tones in the bi215 syllables were reversed (i.e. a novel position), and (4) items where the tone was embedded in a 216 sentence context. As in the study by Perrachione et al. (2011), Sadakata and McQueen (2014)

217 also tested individual aptitude but with a different method. They employed a categorisation task

218 using stimuli from a six step Tone 2 to Tone 3 continuum (created using natural productions of

219 the two tones with the Mandarin vowel /a/ as endpoints and linearly interpolating between these 220 endpoints). Participants were asked to identify if the sound they heard was more like Tone 2 or

221 Tone 3 , and a categorisation slope was obtained for each participant providing a measure of their

222 ability to discriminate this contrast, which is generally found to be the most challenging tone

223 contrast for L2 learners of Mandarin. Participants were grouped according to their slopes, and

224 this grouping was entered as a factor in the analyses of tests of learning, along with the effect of

225 training condition (high-medium-low) and the interaction between factors. For the test with 
226 trained speakers and items, there was no group level effect of variability condition, however

227 there was an interaction between variability and aptitude similar to that reported by Perrachione

228 et al.: Participants with high aptitude benefitted from high variability training, while those with

229 lower aptitude benefitted more from low variability training. For the generalisation tests,

230 participants showed above chance performance in all but the new position condition,

231 demonstrating an ability to generalise their learning of tone across different dimensions.

232 However, they did not demonstrate an overall benefit of higher variability in any of the transfer

233 tests, nor, did variability interaction with aptitude. Note that the overall lack of a high variability

234 benefit is again surprising, particularly for test items with untrained talkers and novel items,

235 since the manipulations in training should specifically work to increase generalisation along

236 these dimensions.

237 In sum, the two studies which have directly compared high and low variability input in

238 training Mandarin tone contrasts have not found the predicted benefit of high variability on

239 generalisation, either when varying just speakers or when varying speakers and items. However,

240 both of these studies found an interaction between participant aptitude and variability condition.

241 The results of these studies thus provide mutually corroborating evidence - using somewhat

242 different training and testing methods - that the ability to learn from high variability input is

243 dependent on learner aptitude, although it should be noted that this interaction was found in a

244 task with untrained speakers in one study (Perrachione et al., 2011), but in a task with trained

245 stimuli in the other (Sadakata \& McQueen, 2014).

246 Why might the ability to benefit from varied training materials depend on participant

247 aptitude? Perrachione et al. (2011) suggest that one reason why low aptitude participants may

248 struggle with multi-speaker input is that the speakers were intermixed during training: This 
249 requires trial-by-trial adaptation to each speaker, which was not required in the corresponding

250 single speaker low variability conditions. This may place a burden on learners (see Mattys \&

251 Wiget, 2011; Nusbaum \& Morin, 1992, for evidence that intermixed multi-speaker stimuli are

252 difficult even for L1 processing and that this interacts with constraints on working memory and

253 attention). To test this, Perrachione et al. (2011) conducted a second experiment in which items

254 from each speaker were presented in separate blocks (as is more common in HVPT). This

255 improved performance during the training task compared with unblocked training for low

256 aptitude learners only, confirming the hypothesis that switching between speakers on a trial-by-

257 trial basis during training interferes with learning for low aptitude learners. On the other hand,

258 Sadakata and McQueen (2014) employed a training paradigm in which speakers were blocked in

259 the high variability condition, yet they still found the interaction with aptitude. However, recall

260 that in their experiment they also manipulated item variability, yet only speakers were blocked

261 by session, not items. Thus, it remains possible that trial-by-trial inconsistency at the level of

262 items could explain some of the greater difficulty of low aptitude learners in their study.

2631.3 The Current Study

264 The fact that neither of the tone training studies found an overall benefit of high over low

265 variability in tone generalisation is surprising in light of the phonetic literature and the

266 predictions of the computational model (Apfelbaum \& McMurray, 2011; Ramscar \& Baayen,

267 2013) mentioned above. Moreover, as the previous authors point out, if it is actually the case that

268 learning from multiple voices is more or less effective for different groups of learners, this has

269 important implications for the design of L2 training tools. For this to be the case, it is important

270 to establish the generalizability of the findings to different contexts and materials, particularly

271 those which are relevant in an L2 learning context. We suggest that what L2 learners are most 
272 interested in developing is their ability to use tone when mapping a word's phonological form to

273 its meaning (and vice versa). In this light, the paradigm used by Sadakata and McQueen (2014)

274 lacks ecological validity in looking only at mapping to abstract tone categories. On the other

275 hand, Perrachione et al. (2011) do train form-meaning mappings, yet, unlike Sadakata and

276 McQueen (2014) they use English pseudo-word stimuli, which has the consequence that learners

277 do not simultaneously have to deal with non-native segments and tones, as in a real world L2

278 learning situation. Furthermore, although there is limited data on the differences between words

279 and non-words in production, it has been noted that non-words may have different properties

280 from real words even within the same language (Scarborough ,2012) and may be more clearly

281 articulated (Hay, Drager \& Thomas, 2013; Maxwell, Baker, Bundgaard-Nielsen \& Fletcher,

282 2015). Thus, using non-words might make stimuli slightly easier to learn than if real words were 283 used.

284 The current training study addresses these issues in a partial replication of the previous 285 work: We use stimuli produced by native Mandarin speakers which are real words in that 286 language. This design choice follows earlier studies such as Wang et al. (1999) using a paradigm 287 in which participants are trained to identify word meaning on the basis of tone. In contrast to the 288 previous studies, we also trained the contrasts between all four tones (six tone contrasts) rather 289 than just three (on the assumption that learners are interested in learning the complete set of 290 contrasts within a particular language). We note that these design choices potentially increase the 291 difficulty of our training materials compared to previous work. A key question was whether 292 these choices would impact the interaction between learner aptitude and the benefits of more 293 variable training materials. 

speaker variability, keeping training items identical across conditions. We also followed

296 Perrachione et al. (2011) in comparing high variability input which was blocked by speaker, with

297 input that was not, making three training conditions: low variability (one speaker), high speakers each presented in separate blocks). Note that our choice to manipulate only talkervariability means that the high variability blocked condition is matched to the low variability condition in terms of trial-by-trial inconsistency, unlike in Sadakata and McQueen (2014) where, even though they blocked by speaker, the high variability condition contained more trial-by-trial variability in terms of items. We predicted that the difficulty of high variability input for lower aptitude participants would be greater in the unblocked condition, thus potentially increasing the likelihood of seeing the predicted interaction between variability and learner aptitude. On the other hand, blocked input is more usual of HVPT (e.g. Iverson, Hazan \& Bannister, 2005; Logan et al. 1991) and may increase the possibility of seeing an overall benefit of speaker variability on generalisation.

We used two perceptual tasks designed to tap individual aptitude. These were adapted

310 from those used in Perrachione et al. (2011) and Sadakata and McQueen (2014). However, while

311 the previous studies grouped participants into one of two categories (high aptitude vs. low

312 aptitude) based on the aptitude score, in the current study they were used as continuous

313 measures. This allowed us to avoid assigning an arbitrary "cut off" for high versus low aptitude

314 groups, and the loss of information which occurs when an underlying continuous variable is

315 turned into a binary measure. Note that the statistical approach used in the current paper (logistic 
316 mixed effect models) allowed us to include continuous predictors and look at their interactions

317 with other factors.

318 A further extension in the current study is that we use several new outcome measures to

319 test learning and generalisation. First, most similar to the task used in Perrachione et al. (2011)

320 was a picture identification task which was a version of the training task (2AFC picture

321 identification) without feedback. Following Perrachione et al. (2011) we included untrained-

322 speaker items, where benefits of speaker variability in training should be most apparent.

323 However, bearing in mind that Sadakata and McQueen (2014) actually found the key interaction

324 with aptitude only in the test with trained stimuli, we also included trained-speaker test items.

325 We also included a second perceptual task which did not involve knowing specific form-

326 meaning mappings and thus had the benefit that it could be conducted both pre- and post- test.

327 This was a three interval oddity task which required participants to pick the odd-one-out after

328 hearing three words spoken aloud, each by a different speaker. Two of the tokens were

329 productions of the same word and the third differed only in the tone (e.g. $b \bar{a}$, Tone $1 ; b \bar{a}$, Tone 1;

$330 b \grave{a}$, Tone 4). Because all three tokens are physically different, it requires the listener to focus on

331 the phonological level ignoring irrelevant acoustic differences. Furthermore, the use of three

332 speakers forces the listener to ignore irrelevant speaker-specific differences, making it especially

333 challenging (Strange \& Shafer, 2008). This task used untrained speakers in every trial, so that

334 every test-item required generalisation to new speakers ${ }^{2}$. In addition, here it was possible to use

335 both trained and untrained items. Note that even though the variability over items is matched

336 across conditions, it is possible that varying speaker specific cues might also thus promote

\footnotetext{
${ }^{2}$ If we wished to use trained speakers, in order to be able to the use the same test with the low variability condition, we would have to use a single speaker across all three test trials. Our pilot work suggested that participants performed at ceiling on a single-speaker version of this task, even at pre-test.
} 
337 generalisation across this dimension. If this is the case, a high variability benefit may be stronger

338 for untrained items than trained items.

339 Finally, we also tested production using a picture naming task at post-test, in which

340 participants were required to name the pictures used in training in Mandarin. We also conducted

341 a word repetition task, which had the benefit that it could also be conducted at pre-test, and that

342 we could use both trained and untrained words (as for the three-interval oddity task discussed

343 above). Although there is evidence HVPT can benefit the production of tones (Wang et al.,

344 2003), there has been no direct examination of whether high variability training materials are

345 more effective than low variability training materials for production. However, more generally in

346 the L2 vocabulary learning literature, training with multiple speakers has been found to lead to

347 better recall in a picture naming task (Barcroft \& Sommers, 2005), suggesting that the HVPT

348 advantage should extend to production measures.

349 In sum, the current experiment assessed whether individuals benefit from high over low

350 variability perceptual training when learning novel L2 tone contrasts, and whether this interacts

351 with learner aptitude. We used measures of aptitude taken from previous studies, but a training

352 paradigm with real Mandarin stimuli embedded in a vocabulary learning task, which trained

353 discrimination of all six Mandarin tone contrasts. Learning and generalisation were measured in

354 multiple tests of both perception and production. In general, the current design increased

355 ecological validity and likely also increased the difficulty of the learning task relative to previous

356 work. It is possible that increasing difficulty could exacerbate differences between learners of

357 different aptitudes, potentially increasing the effect. On the other hand, it is also possible that the

358 increased difficulty might make high variability input much harder for all participants,

359 decreasing or removing the specific benefit of HVPT for high aptitude learners. 
$\begin{array}{lll}360 & 2 & \text { Method }\end{array}$

\section{$361 \quad 2.1 \quad$ Participants}

362 Sixty adults recruited from UCL Psychology Subject Pool participated in the experiment,

363 twenty in each of the three conditions (low variability, high variability, high variability blocked).

364 Participant information is summarised in Table 1. There was no difference between these groups

365 in age, $F(2,57)=1.95, p=.15$. Participants had no known hearing, speech, or language

366 impairments. Written consent was obtained from participants prior to the first session. Each

367 participant was paid $£ 45$ at the end of the study.

368 All participants except three were native English speakers. Of the remaining three, one

369 participant (low variability condition) was a native bilingual of English and Hindi, one participant

370 (high variability condition) was a native French speaker, and one participant (high variability

371 condition) was a native Finnish speaker. Critically, participants had no prior experience of

372 Mandarin Chinese or any other tonal language. On average, participants had learned $2.4(S D=0.8)$

373 languages and the average age for starting to learn the first L2 was 12.6 years $(S D=1.3)$.

374 Ethical approval was given by the UCL Research Ethics Committee with the approval

375 number 6176/002.

\section{$376 \quad 2.2 \quad$ Stimuli}

377 2.2.1 Stimuli used in Training and in the Picture Identification, Three Interval Oddity, Word $378 \quad$ Repetition and Picture Naming Tests

379 These stimuli consisted of 36 minimal pairs of Mandarin words (6 minimal pairs for each

380 of the six tone contrasts generated by the four Mandarin tones). The words in each pair contained

381 the same phonemes, differing only in tone (e.g. māo, Tone 1 [cat] vs. mào, Tone 4 [hat]). All

382 words were picturable and started with a wide range of phonemes (see Appendix A). In order to 
383 examine generalisation across items, half of the word pairs ( 3 per tone contrast) were designated

384 "trained" words and other half were designated "untrained" words. Trained words were

385 encountered in both training and test tasks; untrained words were only encountered in the three

386 interval oddity and word recognition tests.

387 The full set of 72 Mandarin words was recorded by two groups of native Mandarin

388 speakers using a Sony PCM-M10 handheld digital audio recorder. The first group consisted of

389 three female and two male speakers. These stimuli were used in the Training, Word Repetition

390 and Picture Identification tasks. The second group consisted of three new female speakers and

391 two new male speakers. These stimuli were used in the three interval oddity task (making all new

392 speakers in that task). See Table 2 for a summary of the manipulation of item and speaker

393 novelty across the different test tasks, and Table 3 for the tasks in which speakers are

394 counterbalanced.

395 In the low variability condition only one speaker (Trained voice 1) was used in training, 396 and this same speaker was also used as the test voice in the Word Repetition test and for trained

397 items in the picture identification test. In the high variability conditions, four speakers (Trained

398 voice 1 plus three others) were used in training. Only one of these speakers (Trained voice 1)

399 was used in the word repetition test and for trained items in the picture identification test. In all

400 conditions, a further speaker (Untrained voice 1) was assigned to the untrained test items in the

401 picture identification test. The assignment of speakers was rotated across participants, resulting

402 in five counterbalanced versions of each condition (see Table 3). This ensured that any

403 difference found between the low and high variability conditions, and between trained and

404 untrained voices, were not due to idiosyncratic difference between speakers. There was no

405 counterbalancing of speaker in other tasks. 
All words were edited into separate sound files, and peak amplitude was normalized

407

408

409

410

411

412

413

414

416

using Audacity (Audacity team, 2015, http://audacity.sourceforge.net/). Any background noise

was also removed. All recordings were perceptually natural and highly distinguishable as judged by native Chinese speakers. Clipart pictures of the 72 words were selected from free online clipart databases.

\subsubsection{Stimuli used in the Aptitude Tests:}

Pitch Contour Perception Test: Six Mandarin vowels (/a/, /o/, /e/, /i/, /u/, /y/) were repeated in the four Mandarin tones by two male and two female native Mandarin speakers from talker set 2, making 96 stimuli in total. Stimuli were identical across conditions and participants. Categorisation of Synthesised Tonal Continua: Natural endpoints were chosen from a native Mandarin male speaker producing the word 'wan' with both Tone 2 and Tone 3 . A neutral vowel was also recorded by a native male English speaker producing the 'father vowel' /a/. This vowel was edited slightly to remove portions containing creaky voice at the end. The three syllables (wan [Tone 2], wan [Tone 3], /a/) were then manipulated in Praat (Boersma \& Weenink, 2015). All three syllables were normalized to be approximately $260 \mathrm{~ms}$ long using the PSOLA method. The neutral vowel was manipulated to have a flat fundamental frequency (148 $\mathrm{Hz}$ ) and a flat intensity contour $(75 \mathrm{~dB})$. The pitch contours of the two natural endpoints were extracted and a 6-step pitch continuum (Step 1: Tone 2, Step 6: Tone 3) was generated by linearly interpolating between the endpoints. These six pitch contours were then each superimposed on a copy of the neutral vowel using the PSOLA method. Stimuli were identical across participants and conditions.

\subsection{Procedure}


429 (sessions 2-7), and post-test (session 8). Participants were required to complete all eight sessions

430 within two weeks, with the constraint of one session per day at most. The majority of sessions

431 took place in a quiet, soundproof testing room in Chandler House, UCL. The remaining sessions 432 took place in a quiet room in a student house.

433 Participants were given a brief introduction about the aim of the study and told that they

434 were going to learn some Mandarin tones and words. They were explicitly told that Mandarin

435 has four tones (flat, rising, dipping and falling) and that the tonal differences were used to

436 distinguish meanings. The experiment ran on a Dell Alienware 14R laptop with a 14-inch screen.

437 The experiment software was built using a custom-built software package developed at the

438 University of Rochester.

439 The specific instructions for each task were displayed on-screen before the task started.

440 After each task, participants had the opportunity to take a 1-minute break. The tasks completed

441 in each session are listed in Figure 1 and described in more detail below. Note that the Pitch

442 Contour Perception Test and Categorisation of Synthesized Tonal Continua were carried out at

443 the beginning of the first session as they provided the measure of individual aptitude prior to 444 exposure to any Mandarin stimuli. There was no time limit for making responses in any of the

445 tasks. Participants wore a pair of HD 201 Sennheiser headphones throughout the experiment with 446 audio stimuli presented at a comfortable listening level.

447 2.3.1 Individual Aptitude Measures

\subsubsection{The Pitch Contour Perception Test}

This test was based on the work of Wong and Perrachione (2007). Participants heard a

450 tone (e.g. /a/ [Tone 1]), while viewing pictures of four arrows indicating the different pitch

451 contours. Participants clicked on the arrow that they thought matched the tone heard. No 
452 feedback was provided. There were 96 stimuli in total ( 4 speakers $* 4$ tones $* 6$ vowels). This

453 task provided another measure of individual differences in tone perception prior to training.

454 Although Perrachione et al. only conducted this task at pre-test, for consistency with the

455 Categorisation of Synthesised Tonal Continua (described below) we also repeated the test at

456 post-test and conducted analyses to identify whether performance on this task was itself

457 improved as a result of training (see Section 3.2.2).

458

\subsubsection{Categorisation of Synthesised Tonal Continua}

This test was based on Sadakata and McQueen (2014). Participants first practiced

listening to Tone 2 and Tone 3 while viewing the corresponding picture of an arrow depicting the

461

pitch change. Each tone was repeated 10 times. In each test trial, participants then decided

462 whether the sound they heard was closer to Tone 2 or Tone 3 by clicking on the corresponding

463 arrow. No feedback was provided. The speech continua consisted of 6 steps (Step 1: Tone 2,

464 Step 6: Tone 3) with each step repeated 10 times per block. Participants completed two blocks,

465 with an optional 1 minute break in the middle, resulting in 120 trials in total. This task provided a

466 measure of individual differences in tone perception prior to training. In line with Sadakata and

467 McQueen's procedure, participants completed the task both before and after training and we

468 conducted analyses to explore whether there was improvement from pre to post-test (Section

$4693.2 .1)$.

470

\subsubsection{Training Task}

471

Participants completed the training task in Session 2-7. On each trial, participants heard a

472 Mandarin word and selected one of two candidate pictures displayed on the computer screen.

473 The two pictures always belonged to the same minimal pair. Feedback was provided about

474 whether the answer was correct (a green happy face appeared) or incorrect (a red sad face

475 appeared). If the correct choice was made, a picture of a coin also appeared in a box on the left- 
476 hand side of the screen, with the aim of motivating participants to try to earn more coins in each

477 subsequent session of training. After that, everything but the correct picture was removed from

478 the screen and the participant heard the correct word again. In the lower right corner of the

479 screen a trial indicator of $\mathrm{X} / 288$ was displayed where $\mathrm{X}$ indicated the number of trials completed.

480 This tool helped participants to keep track of their performance (see Figure 2).

481 There were 18 picture/word pairs used. Each word was used as the target four times.

482 Thus, each picture pair appeared eight times, resulting in 288 trials per session. Participants were

483 assigned to one of the following conditions: low variability, high variability and high variability

484 blocked (with the assignment of speakers counterbalanced - see Table 3). Each training session

485 lasted for approximately 30 minutes.

486 In the low variability condition, only one speaker was used. In the high variability

487 conditions, four speakers were used. For each participant, each of their six training sessions was

488 identical. In the high variability condition without blocking, all of the speakers were heard in

489 each of the training sessions, with the order randomized so that speaker varied from trial to trial.

490 In contrast, in the high variability blocked condition, from Day 1 to Day 4 of training (i.e.,

491 Session 2-5), only one speaker was involved on each day's training session, (with the trained

492 speaker that was used in the test tasks (e.g. F1 for Version 1) always occurring on Day 3 (i.e.,

493 Session 4)); on Days 5 and 6 of training (i.e., Sessions 6 and 7), participants heard all four

494 speakers, each in a separate block, with each word being repeated twice in each voice on these

495 days. In all three conditions, the order of items was randomized within each session.

$496 \quad 2.3 .3 \quad$ Perceptual Tests

497

498

499

\subsubsection{Three Interval Oddity Test (pre- post test)}

This task required participants to identify the odd one out (i.e. the stimulus with a different tone) from a choice of three Mandarin words, each spoken by a different speaker. Four 
500 untrained speakers were used ( 3 female, 1 male). Each trial used one of the 36 minimal pairs

501 from the main stimuli set (18 trained pairs, 18 untrained pairs). Preliminary work suggested that

502 trials differed in difficulty depending on whether the "different" stimulus was spoken by the

503 single male speaker, or one of the three female speakers. We therefore ensured that there were

504 equal numbers of the following trial types: (i) "Neutral" - all three words were spoken by female

505 speakers (ii) "Easy" - the "different" word was spoken by a male speaker and the other two were 506 spoken by female speakers; (iii) "Hard" - the "different" word was spoken by a female speaker

507 and the other two were spoken by one male speaker and one female speaker. Each of the words

508 in the minimal pair was used once as the target ("different") word, making 72 trials in total.

509 During the task, three frogs were displayed on the screen. Participants heard three words

510 (played with ISIs of 200ms) and indicated which word was the odd one out by clicking on the

511 appropriate frog, which could be in any of the three positions. They could not make their

512 response until all three words had been heard, at which point a red box containing the instruction

513 "Click on the frog that said the different word" appeared at the bottom of the screen. No

514 feedback was provided. Participants completed this task twice - once in the pre-test, and once in

515 the post-test.

\subsubsection{Picture Identification Test (post- only test)}

This task was the same as the training task with the following changes. Firstly, each word speaker (Untrained voice 1), making 72 trials in total. Secondly, no feedback was given. This task was completed only in the post-test. 
521

522

523

524

525

526

527

528

529

530

531

532

533

534

535

536

537

538

539

540

541

542

543

544

\subsubsection{Production Test}

\subsubsection{Word Repetition Test (pre-post test)}

All seventy-two Mandarin words from the main stimulus set (18 trained pairs, 18

untrained pairs) set were presented one at a time in a randomised order. They were always spoken by the same speaker and this speaker was also used in their training stimuli (training voice 1; see Table 3). After each word, two seconds of white noise was played. This was included to make sure that participants had to encode the stimulus they were repeating and could not access the information in echoic storage (Flege, Takagi \& Mann, 1995). Participants were instructed to listen carefully to the word and then to repeat the word aloud after the white noise.

Verbal responses were digitally recorded and were later transcribed and rated by native speakers of Mandarin (see Section 3.5.1). This task was completed once in the pre-test and once in the post-test.

\subsubsection{Picture Naming Test (post-only test)}

All 36 pictures from the training words were presented in a randomised order.

Participants were instructed to try to name the picture using the appropriate Mandarin word. Verbal responses were recorded and were later transcribed and rated by native Mandarin speakers (see Section 3.5.1). This task was completed only in the post-test.

\subsubsection{Other tasks}

\subsubsection{English Introduction Task}

This task was included in the batch of tasks administered at pre-test in case the meaning of some pictures were ambiguous (not all items were concrete nouns - e.g. "to paint"). Participants saw each of the 36 pictures from the training set presented once each in a random order and heard the corresponding English word. No response was recorded. Participants completed this task only once, at the end of the pre-test session. 
545

546

547

548

549

550

551

552

553

554

555

556

557

558

559

560

561

562

563

564

565

566

567

568

\subsubsection{Questionnaires}

Participants completed a language background questionnaire after the experiment.

Participants were asked to list all the places they had lived for more than 3 months and any

languages that they had learned. For each language the participant was asked: (a) to state how

long they learned the language for and their starting age; (b) to rate their own current proficiency of the language.

\section{$3 \quad$ Results}

\subsection{Statistical Approach}

Three different sets of frequentist analyses are reported. First, we conducted the analysis on two individual measures Categorisation of Synthesized Tonal Continua (Section 3.2.1) and Pitch Contour Perception Test (Section 3.2.2). The primary aim of these analyses was to ensure that the three groups did not differ at pre-test, however we also looked for possible differences at post-test. Second, separate analyses are reported on data from the tests administered pre- and post- training (i.e. Word Repetition task (Section 3.5.2) and Three Interval Oddity task (Section 3.4.1)), the data collected during Training (Section 3.3) and the data from the two tasks administered only at post-test (i.e. the Picture Identification task (Section 3.4.2) and Picture Naming task (Section 3.5.3). These analyses explored the effects of our experimentally manipulated conditions on the various measures of Mandarin tone learning. Third, analyses were conducted exploring the role of aptitude in each of these tasks (Section 3.6). Specifically, we wanted to see whether aptitude interacted with variability-condition in predicting the benefits of training, in line with the predictions of previous research (Perrachione et al., 2011; Sadakata \& McQueen, 2014).

Except where stated, analyses used logistic mixed effect models (Baayen, Davidson, \& Bates, 2008; Jaeger, 2008; Quené \& Van den Bergh, 2008) using the package lme4 (Bates, 
569 Maechler, \& Bolker, 2013) for the R computing environment (R Development Core Team,

570 2010). Logistic mixed effect models allow binary data to be analysed with logistic models rather

571 than as proportions, as recommended by Jaeger (2008). In each of the analyses, the factor

572 variability-condition has three levels (low variability [LV], high variability $[\mathrm{HV}]$, and high

573 variability blocked [HVB]) which we coded into two contrasts with LV as the baseline (LV

574 versus $\mathrm{HV}, \mathrm{LV}$ versus $\mathrm{HVB}$ ). An exception to this is the training data, where a model containing

575 all three conditions would not converge and we took a different approach, as described in Section

576 3.3. We also included the interactions between these contrasts and the other factors. We used

577 centred coding which ensured that other effects were evaluated as averaged over all three levels

578 of variability-condition (rather than the reference level of LV3). Similarly, for the Three Interval

579 Oddity task, we included a trial-type factor. The purpose of this was to control for the fact that

580 participants were likely to find some trial types easier than others due to the gender of the

581 speakers producing the stimuli (see Section 2.3.3.1). We therefore coded a factor trial-type with

582 three levels (neutral, easy, hard- see method) and included contrasts with neutral ("neutral versus

583 easy" and "neutral versus hard") using centered coding. In order to perform the analysis

584 comparing pre- and post-test performance, test-session was coded as a factor with two levels

585 (pre-test/post-test) with "pre-test" set as the reference level. This allowed us to look at the

586 (accidental) possible differences between the experimental conditions at the pre-test stage, as

587 well as whether post-test performance differed from this baseline. All other predictors, including

588 both discrete factor codings with two levels (item-novelty in the Word Repetition and Three

589 Interval Oddity tasks, and voice-novelty in the Picture Identification task) and numeric predictors

590 (training-session) in the Training data analyses and the individual difference measures in the

\footnotetext{
${ }^{3}$ This differs from the default coding of contrasts in the lme4 package. It was achieved by replacing the three-way
} factor "condition" with two centred dummy variables and using the main fixed effects from the output of this model. 
591 models reported in Section 3.6), were centred (i) to reduce the effects of collinearity between

592 main effects and interactions, and (ii) so that the main effects were evaluated as the average

593 effects over all levels of the other predictors (rather than at a specified reference level for each

594 factor). We automatically put experimentally manipulated variables and all of their interactions

595 into the model, without using model selection (except for "trial-type" in the Three Interval

596 Oddity task which works as a control factor and for this factor we only used its main effect and

597 the interaction with test-session). However, we did not inspect the models for all main effects

598 and interactions. Instead, we report the statistics which were necessary to look for accidental

599 differences at pre-test, and those related to our hypotheses. We aimed to examine whether the

600 training improved participants' performance on both untrained items and untrained voices and

601 whether such improvement was modulated by their individual aptitudes. Participant is included

602 as a random effect and a full random slope structure was used (i.e., by-subject slopes for all

603 experimentally manipulated within-subject effects (test-session, voice-novelty, item-novelty) and

604 interactions, as recommended by Barr, Levy, Scheepers, and Tily (2013). In some cases the

605 models did not converge and in those cases correlations between random slopes were removed.

606 Models converged with Bound Optimization by Quadratic Approximation (BOBYQA

607 optimization; Powell, 2009). R scripts showing full model details can be found here:

608 https://osf.io/wdh8a/?view only=ad8455b30b2e4271aaa4cc55fc94a40f

609 In addition to the frequentist analyses, in order to aid interpretation of key null results we

610 also included Bayes factor analyses. Our approach for these is described within the relevant

611 section (Section 3.7).

$612 \quad 3.2 \quad$ Individual Aptitude Tasks 


\section{3.2.1 The Pitch Contour Perception Test}

614 The predicted variable was whether a correct response was given $(1 / 0)$ on each trial. The 615 predictors were the contrasts between variability-conditions (LV versus HV; LV versus HVB) 616 and test-session (pre-test, post-test). There was no significant difference between the LV and HV 617 groups $(\beta=-0.35, S E=0.26, z=-1.38, p=0.17)$ or between the LV and HVB groups $(\beta=0.17$, $618 S E=0.26, z=0.66, p=0.51)$ at pre-test on this measure. Participants showed significant

619 improvement after training $(\beta=0.21, S E=0.05, z=4.13, p<0.001)$, which can be seen in 620 Figure 3.

621 Thus, the three participant groups did not differ in their pre-test performance and the 622 groups showed equivalent improvement from pre- to post- test. Given that this measure is 623 affected by training, we used participants scores at pre-test as our measure of individual 624 differences in the analyses reported in Section 3.6..

\section{3.2.2 Categorisation of Synthesised Tonal Continua}

626 We estimated individual's performance on the Categorisation of Synthesised Tonal

627 Continua task following Sadakata and McQueen (2014). We used the Logistic Curve Fit function 628 in SPSS to calculate a slope coefficient for each participant (Joanisse, Manis, Keating \& 629 Seidenberg, 2000). The slope (standardized $\beta$ ) indicates individual differences in tone perception. 630 The smaller the slope, the better the performance. Sadakata and McQueen, removed data from 631 participants with a slope measuring greater than 1.2. Using this threshold $43 / 60$ participants 632 failed the threshold in the current study. This is consistent with the observation that most of the 633 participants were not able to consistently categorise the endpoints of the continua, indicating that 634 this was not a good test of aptitude. We do not report further analyses involving this aptitude 
635 variable however they can be found in the supplemental materials

636 (https://osf.io/wdh8a/?view_only=ad8455b30b2e4271aaa4cc55fc94a40f).

\section{$637 \quad 3.3 \quad$ Training}

638 A model containing data from all three conditions did not converge; however two

639 separate models, one including the LV and HV conditions, and the other the LV and HVB

640 conditions (with condition as a factor with two levels), did converge. In each case the predicted

641 variable was whether a correct response was given $(1 / 0)$ on each trial. The predictors were the

642 numeric factor training-session (1:6) and the factor variability-condition which had two levels

643 (Model 1: LV versus HV; Model 2, LV versus HVB). The mean accuracy is displayed in Figure 6444.

645 In both models, there was an effect of training-session (Model 1: $\beta=0.49, S E=0.04, z=11.52$, $646 p<.001$; Model 2: $\beta=0.53, S E=0.04, z=12.17, p<.001)$ : Participants' performance increased 647 significantly over time, with additional training sessions. Overall, the LV group performed better 648 than both the HV group $(\beta=-0.79, S E=0.16, z=-5.03, p<.001)$ and the HVB group $(\beta=-$ $6490.83, S E=0.32, z=-2.61, p<.01)$. However, the LV versus HV contrast was also modulated by 650 an interaction with test-session $(\beta=-0.19, S E=0.04, z=-4.59, p<.001)$, as was the LV versus 651 HVB contrast $(\beta=-0.35, S E=0.08 z=-4.33, p<.001)$. From Figure 4 it can be seen that the 652 LV and the HVB group did not differ in the first session (i.e. where they get identical input) but 653 the difference gradually increased over the next few sessions. For the LV and the HV group, they 654 differed starting from the first session and this difference continued to increase throughout 655 training.

$656 \quad 3.4 \quad$ Perceptual tests 


\section{3.4.1 Three Interval Oddity Task}

658 The predicted variable was whether a correct response was given $(1 / 0)$ on each trial. The

659 predictors were test-session (pre-test, post-test), variability-condition (LV versus HV, LV versus

660 HVB), trial-type (neutral versus easy, neutral versus hard) and item-novelty (trained item,

661 untrained item). The mean accuracy is displayed in Figure 5.

662 At pre-test, there was no significant difference between the LV and HV groups $(\beta=$ -

$6630.002, S E=0.14, z=-0.01, p=.99)$ nor between the LV and HVB groups $(\beta=0.12, S E=0.14, z$

$664=0.86, p=.39)$, suggesting that the groups started at a similar level. However, performance with

665 the "untrained" was significantly greater than performance on the "trained" items at pre-test $(\beta=$

$666-0.31, S E=0.06, z=-4.95, p<0.01)$, suggesting incidental differences between item sets. As

667 expected, at pre-test participants performed significantly better on "easy" trials (where the target

668 speaker had a different gender) than "neutral" trials (where all three speakers had the same

669 gender, $\beta=0.40, S E=0.08, z=5.09, p<0.01)$ and "neutral" trials were marginally easier than

670 "hard" trials (where one of the foil speakers had the odd gender out, $\beta=-0.14, S E=0.08, z=$ -

$671 \quad 1.81, p=0.07)$

672 Overall, participants' performance increased significantly after training $($ Mpre $=0.59$,

673 SDpre $=0.21$, Mpost $=0.66$, SDpost $=0.19, \beta=0.31, S E=0.05, z=6.54, p<.001)$. The

674 interaction between test-session and item-novelty was not significant $(\beta=0.14, S E=0.09, z=$

$6751.49, p=.14)$, suggesting no evidence that training had a greater effect for trained words than for

676 untrained words. Critically, there was no interaction with test-session for either the contrast

677 between the LV versus the HV conditions $(\beta=-0.01, S E=0.12, z=-0.12, p=.90)$ or the

678 contrast between the LV versus the HVB conditions $(\beta=0.01, S E=0.12, z=0.11, p=.91)$ and

679 they were not qualified by any higher level interactions with item-novelty (LV versus HV: $\beta=$ - 
$0.1, S E=0.22, z=-0.64, p=0.52 ; \mathrm{LV}$ versus HVB: $\beta=0.13, S E=0.22, z=0.57, p=0.57)$

681 This suggests no evidence that the extent to which participants improved on this task between

682 pre and post-test differed according to variability-conditions, or that this differed for trained

683 versus untrained items.

684 Although not part of our key predictions, we also looked to see if there was evidence that 685 participants improved more with the easier or harder trials. In fact, the interaction between test686 session and the contrast between "easy" and "neutral" was significant $(\beta=-0.27, S E=0.11, z=$ $2.39, p=.02)$ while the contrast between "neutral" and "hard" was not $(\beta=0.12, S E=0.11, z=$

$1.06, p=.29)$. This was due to the fact that there was improvement for "neutral" (Mpre $=0.57$, SDpre $=0.14$, Mpost $=0.65$, SDpost $=0.15)$ and "hard" trials $($ Mpre $=0.54$, SDpre $=0.16$,

Mpost $=0.65$, SDpost $=0.15)$ but not for "easy" trials $($ Mpre $=0.66$, SDpre $=0.16$, Mpost $=0.68$,

SDpost $=0.15)$.

\section{3.4.2 Picture Identification}

The predicted variable was whether a correct response was given (1/0) on each trial. The

694 predictors were the factor voice-novelty (Trained voice, Untrained voice) and the factor

variability-condition which had two contrasts (LV versus HV, LV versus HVB). The mean

accuracy is displayed in Figure 6.

697

There was a main effect of voice-novelty $(\beta=1.07, S E=0.16, z=6.53, p<.001)$

reflecting higher performance in trials with trained voices. Although participants in the LV group

699 performed better than those in the HV group $(\beta=-0.71, S E=0.32, z=-2.23, p=.03)$, there was

700 no significant difference between the LV and the HVB group $(\beta=-0.14, S E=0.32, z=-0.44, p$

$701=.66)$ and there was a significant interaction between voice-novelty and both the LV-HV contrast

$702(\beta=-1.19, S E=0.35, z=-3.43, p<.01)$ and the LV-HVB contrast $(\beta=-1.11, S E=0.36, z=$ - 
$7033.08, p<.01)$. Breaking this down by variability-condition: for each condition there was

704 significantly better performance with trained than untrained voices (LV: $\beta=1.83, S E=0.29, z$

$705=6.42, p<0.001 ; \mathrm{HV}: \beta=0.64, S E=0.23, z=2.86, p<0.01 ; \mathrm{HVB}: \beta=0.73, S E=0.26, z=$

$7062.82, p<0.01$ ), indicating greater ease with the familiar voice. Breaking down by voice-novelty:

707 For the trained voice, performance was higher in the LV condition than in either the HV or HVB

708 conditions, although this was only significant for the LV versus HV contrast (LV versus HV: $\beta=$

$709-1.30, S E=0.44, z=-2.97, p<0.01$; LV versus HVB: $\beta=-0.70, S E=0.45, z=-1.55, p=0.12$ ).

710 Importantly, for untrained voices, neither of the contrasts between conditions was significant

711 (LV versus HV: $\beta=-0.12, S E=0.26, z=-0.45, p=0.65 ; \mathrm{LV}$ versus $\mathrm{HVB} \beta=0.41, S E=0.27, z$

$712=1.51, p=0.13$ ), indicating no evidence for greater generalisation following high variability

713 training.

$714 \quad 3.5 \quad$ Production tests

715 3.5.1 Coding and inter-rater reliability analyses

716 The same methods were used for both production tests. The files were combined into a

717 single set, along with the 360 stimuli which were used in the experiment (and which were

718 produced by native Mandarin speakers). The latter items were included in order to examine

719 whether the raters were reliable. All stimuli were rated by two raters: Rater 1 was the first author

720 and Rater 2 was recruited from the UCL MA Linguistics program and was naïve to the purposes

721 of the experiment. Raters were presented with recordings in blocks in a random sequence (blind

722 to test-type, condition, whether the stimulus was from pre-test or post-test and whether it was

723 produced by a participant or was one of the experimental stimuli). For each item, raters were

724 asked to (i) identify the tone, (ii) give a rating quantifying how native-like they thought the

725 pronunciation was compared (1-7 with 1 as not recognizable and 7 as native speaker level), and

726 (iii) transcribe the pinyin (segmental pronunciation) produced by the participants. 
If there was no sound or the tone was unrecognizable, the rater coded 0 when identifying

728 the tone. Data from these trials were removed from the dataset before analyses were conducted.

729 In addition, all of the data from one participant was removed from the analyses due to bad

730 recording quality resulting from a technical error. In total, this resulted in $3.38 \%(359 / 10620)$ of

731 production trials being removed from analysis (Word Repetition: Pre-test 1.98\% (84/4248); Post-

732 test 3.72\% (158/4248); Picture Naming 5.51\% (117/2124)). Three measurements were taken

733 from the production tasks: mean accuracy of tone identification (Tone accuracy), mean tone

734 rating (Tone rating) and mean accuracy of production in pinyin (derived by coding each

735 production as correct $(1=$ the entire string is correct $)$ or incorrect $(0=$ at least one error in the

736 pinyin)). As a first test of rater reliability, performance with the native speaker stimuli was

737 examined- these were near ceiling: Rater 1: Tone accuracy $=98 \%$, Tone rating $=6.7$, Pinyin

738 accuracy $=80 \%$; Rater 2: Tone accuracy $=87 \%$, Tone rating $=6.5$, Pinyin accuracy $=80 \%$ ).

739 Furthermore, for the remaining data (i.e. the experimental data) inter-rater reliability was

740 examined for all three measures for the two production tasks. For the binary measures (Tone

741 accuracy and Pinyin accuracy), kappa statistics were calculated using the "fmsb" package in R

742 (Cohen, 2014). For the Word Repetition data, for Tone accuracy kappa $=0.39$ ("fair

743 agreement"), and for Pinyin accuracy kappa = 0.33 ("fair agreement"; Landis \& Koch, 1977).

744 For the Picture Naming test, for Tone accuracy kappa $=0.67$ ("substantial agreement") and for

745 Pinyin accuracy kappa $=0.53$ ("moderate agreement"); For the Tone rating, the package "irr" in

746 R was used to assess the intra-class correlation (McGraw \& Wong, 1996) based on an average-

747 measures, two-way mixed-effects model. For Word Repetition, $I C C=0.22$ and for Picture

748 Identification $I C C=0.37$; according to Cicchetti (1994), values less than .40 are regarded as

749 "poor". Given this, we do not include analyses with Tone Rating as the dependent variable 
750 (though these data are included in the data set

751 https://osf.io/wdh8a/?view_only=ad8455b30b2e4271aaa4cc55fc94a40f). All of the analyses

752 presented in Sections 3.5.2 and 3.5.3 were based on Rater 2 (the naive rater).

753 3.5.2 Word Repetition

754

755

756

757

758

759

760

761

762

763

764

765

766

767

768

769

770

771

772

773

\subsubsection{Tone accuracy}

The predicted variable was whether a correct response was given (1/0) on each trial (as identified by the coder). The predictors were test-session (pre-test, post-test), variabilitycondition (LV versus HV, LV versus HVB) and item-novelty (trained, untrained). The mean accuracy, split by test-session and training condition, is shown in Figure 7

At pre-test, there was no significant difference between the LV and the HV group $(\beta=$ $0.01, S E=0.18, z=0.06, p=.95)$ nor between the LV and the HVB group $(\beta=0.11, S E=0.18$ $z=0.64, p=.53)$, suggesting the groups started at a similar level. There was also no difference between trained and untrained words at pre-test $(\beta=-0.02, S E=0.07, z=0 .-0.26, p=0.80)$. Across the three groups, participants' performance increased significantly after training $($ Mpre $=0.71$, SDpre $=0.09$, Mpost $=0.79$, SDpost $=0.09, \beta=0.40, S E=0.08, z=5.29, p<$ .001). There was no significant difference in the improvement for trained and untrained items (word-type by test-session interaction: $\beta=0.13, S E=0.10, z=1.22 p=.22$ ). Critically, the interactions between the variability contrasts and test-session were not significant (LV versus $\mathrm{HV}: \beta=-0.10, S E=0.18, z=-0.55, p=.58$; LV versus HVB: $\beta=-0.11, S E=0.18, z=-0.62, p=$ .54), and they were not qualified by any higher level interactions with item-novelty (LV versus $\mathrm{HV}: \beta=0.15, S E=0.25, z=0.61, p=.54$; LV versus HVB: $\beta=-0.31, S E=0.26, z=-1.21, p=$ .23). This suggests there is no evidence that participants' improvement in their production of tones was affected by their variability-condition, or that this differed for trained versus untrained items. 


\subsubsection{Pinyin accuracy}

775

The predicted variable was whether the participants produced the correct string of

776

777

778

779

780

781

782

783

784

785

786

787

788

789

790

791

792

793

794

795

796

797

phonemes (1/0) in each trial (as determined by Rater 2). The predictors were test-session (pre-

test, post-test), variability-condition (LV versus HV, LV versus HVB) and item-novelty (trained, untrained). Mean pinyin accuracy is displayed in Figure 8.

At pre-test, there was no significant difference between the LV and the HV group $(\beta=$ $0.01, S E=0.11, z=-0.11, p=.91)$ nor between the LV and the HVB group $(\beta=-0.03, S E=$ $0.11, z=-0.24, p=.81$ ), suggesting that the groups started at a similar level. However, participants did better on untrained words than trained words at pre-test $(\beta=0.21, S E=0.07, z=$ $3.11, p<.01)$, suggesting potential accidental differences in these items. Participants showed significant improvement after training $($ Mpre $=0.54$, SDpre $=0.09$, Mpost $=0.58$, SDpost $=0.19$, $\beta=0.15, S E=0.05, z=3.38, p<.01)$. However, there was no evidence that different variability conditions resulted in different amounts of improvement (test-session by LV versus HV: $\beta=$ 0.05, $S E=0.11, z=0.46, p=.65$; test-session by LV versus HVB: $\beta=-0.12, S E=0.11, z=-$ $1.08, p=.28)$ or any interaction between variability condition, test-session and item-novelty (LV versus $H V: \beta=0.11, S E=0.22, z=0.51, p=.61$; LV versus HVB: $\beta=-0.14, S E=0.22, z=$ $0.64, p=.52)$. This suggests there is no evidence that participants' improvement in pinyin accuracy was affected by their variability-condition, or that this differed for trained versus untrained items.

\subsubsection{Picture Naming}

\subsubsection{Tone accuracy}

The predicted variable was whether a correct response was given (1/0) on each trial (as identified by the coder). There was only one predictor, variability-condition (LV versus HV, LV versus HVB) for both models. The descriptive statistics are displayed in Figure 9. 

group $(\beta=-0.34 S E=0.19, z=-1.81, p=0.07)$ and the HVB group $(\beta=-0.10, S E=0.19, z=$ -

$8000.52, p=.61$. This suggests there is no evidence that participants' ability to produce the tones

801 accurately differed according to their variability-condition.

802

\subsubsection{Pinyin Accuracy}

803 The predicted variable was whether the participants produced the correct string of phonemes

$804(1 / 0)$ in each trial and there was a single predictor variability-condition (LV versus HV, LV

805 versus HVB). For both models there was no significant difference between variability conditions

806 (LV versus HV: $\beta=0.09, S E=0.23, z=0.41, p=0.68$; LV versus HVB: $\beta=0.12, S E=0.23, z=$

$8070.51, p=0.61)$. This suggests there is no evidence that participants' pinyin accuracy differed 808 according to their variability-condition.

$809 \quad 3.6 \quad$ Analyses with Individual Aptitude

810 In order to look at the effect of learner aptitude and the interaction between this factor

811 and variability condition, we first calculated the mean accuracy at pre-test on the Pitch Contour

812 Perception Test for each participant. This score (scaled by a factor of 10, so that each one unit

813 increase in aptitude corresponded to a 10\% higher performance in the Pitch Contour Perception

814 test) was centered and used as a continuous predictor (aptitude) and added to each of the models

815 reported above. In addition, we added the interaction between this factor and key experimental

816 factors (see Table 4). Based on Perrachione et al. (2011) and Sadakata and McQueen (2014), for

817 our measures of tone-learning, high variability should benefit high aptitude participants only,

818 while low variability would benefit low aptitude participants only. In our design, we used a

819 continuous measure of individual ability rather than a binary division of high and low variability.

820 We therefore predicted a stronger positive correlation between aptitude and amount of learning

821 in the high variability condition than in the low variability condition. In the tests administered 
822 only post training (i.e. Picture Identification and Picture Naming) this would show up as an

823 interaction between aptitude and condition. In the models for the pre- and post-test data (i.e.

824 Three Interval Oddity and Word Repetition) this would show up as a three-way interaction

825 between condition, test-session and aptitude. We also looked at the interactions between these

826 factors and voice-novelty (Picture Identification) and item-novelty (Three Interval Oddity and

827 Word Repetition). Note that there are no clear directional hypotheses here: Perrachione et al.

828 (2011) found the interaction in a test with untrained voices and trained items, and Sadakata and

829 McQueen (2014) found the interaction in a test with trained voices and trained items. For

830 training, in principal both the two-way interaction of aptitude by condition and the three-way

831 interaction of aptitude by condition by training-session are of interest. However, it was not

832 possible to fit a converging model containing the three-way factor ${ }^{4}$.

833 Each model reported in Table 4 contained all the fixed effects included in the original

834 models in addition to the fixed effects listed in the table (note that to avoid convergence issues

835 due to over complex models, we did not attempt to include the complete set of interactions for

836 every combination of experimental variables with aptitude - only those for which we had

837 predictions). We attempted to have full random effects structure for these fixed effects however

838 in some cases we had to remove correlations between slopes due to problems with convergence

839 and for one of the models with the training data we had to remove the random slope for training

840 session). Note that we don't include models for the pinyin measures, since our measure of

841 aptitude is relevant to tone learning only. For each of the new models we first confirmed that

842 adding in the new effects and interactions with the individual measures did not change any of the

\footnotetext{
${ }^{4}$ This was the case even if we split the data into two models, as we did in Section 3.3.
} 
843 previously reported patterns of significance for the experimental effects (see script

844 https://osf.io/wdh8a/?view only=ad8455b30b2e4271aaa4cc55fc94a40f) for full models.

845 The results are shown in Table 4. Aptitude is a positive predictor of performance in each

846 of the tests and in training, with p-values significant or marginal in each case. However there was

847 no interaction between aptitude and any other factor. Thus, there was no evidence that this

848 measure of aptitude correlated with participants ability to benefit from training (no interaction

849 with test-session), nor - critically for our hypothesis - did this differ by training condition (no

850 interaction with condition or with test-session by condition).

851 Although the analyses use a continuous measure of Pitch Contour Perception Test, for the

852 purposes of visualisation, Figure 10 (Three Interval Oddity task and Training task), Figure 11

853 (Picture Naming and Picture Identification) and Figure 12 (Word Repetition) use the mean

854 accuracy for participants split into aptitude groups using a median split based on their Pitch

855 Contour Perception Test score.

856 In sum, participants with higher aptitude measures were better at the tasks, but there is no

857 evidence either that this affected their improvement due to training, or, critically, their ability to

858 benefit from the different variability exposure sets.

\section{$859 \quad 3.7 \quad$ Bayes Factor Analyses}

860 In the analyses reported above, we did not find evidence - in any of our tests - for either

861 of two key hypotheses: (1) the hypothesis that training with multiple speakers leads to greater

862 generalization to new speakers than training with a single speaker or (2) the hypothesis that there

863 is an interaction between the variability of the training materials and participant aptitude, such

864 that higher aptitude participants benefit more from training with multiple speakers while lower

865 aptitude participants benefit more from training with a single speaker. However, there is a 
866 difficulty in interpreting these null results since a non-significant result $(\mathrm{p}>.05)$ does not tell us

867 whether we have evidence for the null, as opposed to no evidence for any conclusion at all, or

868 even evidence against the null. Thus, we should not reduce our confidence in either of our

869 hypotheses on the basis of the null results reported above (despite the fact that reducing

870 confidence in a theory following non-significant results is common practice) - see Dienes (2014)

871 for discussion. An alternative statistic is a Bayes Factor, which are used to assess the strength of

872 evidence for one theory (H1) over another (the null hypothesis). We therefore supplemented the

873 analyses above by computing Bayes factors for contrasts relating to these two key hypotheses.

874 These are reported in sections 3.7.1 and 3.7.2 below.

875 3.7.1 H1: Greater generalization - to novel voices and in production - in the multiple speaker conditions (HV and HVB) than in the low variability condition (LV) We aimed to compute Bayes Factors comparing this hypothesis to the null for each of our

878 data sets. To have maximum evidence, we pool the HV and HVB conditions and contrast this 879 with the LV condition. For the post-tests we are interested in the evidence for a main effect of

880 this contrast. For the pre-post tests, we are interested in the interaction between this contrast and 881 session. To further maximize evidence, for the Three Interval Oddity test and Word Repetition

882 tests we look at trained and untrained items combined (since both types of item involve

883 generalisation to an untrained voice and thus should benefit from high variability training),

884 however in the Picture Identification test we excluded trained voice test items, since the benefit 885 of high variability training was not predicted for these items. For the production measures, we 886 are interested in whether there is a high variability benefit for our tone learning measure and our 887 pinyin measure (the latter given that Barcroft and Sommers, 2014, found a benefit of multi888 speaker training in their vocabulary recall task). 
890 (2018). To compute a Bayes factor $(B)$ it is necessary to have both a model of the data and a

891 model of H1. The model of the data is an estimate of the mean difference for the contrast in

892 question, and of the standard error. Here, we get these estimates by running logistic mixed

893 models and taking the betas and standard errors for the relevant coefficients (note that this allows

894 us to meet normality assumptions by continuing to work within log-odds space). The models we

895 ran here were similar to the previous analyses but with variability-condition coded as a centered

896 contrast between LV and the HV+HVB conditions, and other factors combined/excluded as

897 described in the previous paragraphs. The full set of models is in

898 https://osf.io/wdh8a/?view_only=ad8455b30b2e4271aaa4cc55fc94a40f.

899 We model H1 using a half-normal distribution with a mode of 0 and a standard deviation

$900 x$ which is set to be a rough estimate of the predicted difference for this contrast. This allows for

901 possible effects between 0 and twice the predicted effect, with values closer to 0 being more

902 likely (Dienes, 2014).

903 In the absence of any prior data using sufficiently similar materials, and since we did not

904 wish to use unprincipled default values, we estimated $x$ for each contrast using the scale and/or

905 values from elsewhere in the data (see Dienes 2014, 2015 for a related approach). Specifically,

906 for each of the cases where we predicted a main effect (Picture Identification and Picture

907 Naming), we set $x$ as the difference between the grand mean (the Intercept - since we use a

908 centered coding) and an estimate of minimal possible performance on the task. The logic is as

909 follows ${ }^{5}$ : The maximum difference between conditions is seen if low variability participants

910 show baseline performance and high variability participants show performance greater than

\footnotetext{
${ }^{5}$ Further details of the logic of these computations is spelt out in the script available at
} https://osf.io/wdh8a/?view_only=ad8455b30b2e4271aaa4cc55fc94a40f 
911 baseline. In this case, if performance on this test is $p$ (so the grand mean is $\bar{p}$ ) and the baseline is

$912 b$, the difference in $p$ between the two conditions will be equal to: $2(\bar{p}-b)$. This gives us an

913 estimate of the maximum value of $x$; since we are using a half normal distribution with a mean of

914 zero, we assume the maximum value is equal to approximately $2 S D$, so we can set our estimate $x$

915 of the standard deviation to be equal to half of this value (i.e. $x=\bar{p}-b$.). Baseline performance

916 depends on the task: for the $2 \mathrm{AFC}$ Picture Identification task it is chance $(50 \%=0 \mathrm{in} \log$ odds

917 space); for the Picture Naming, tone measure, we assume a $1 / 4$ chance of identifying the correct

918 one $(25 \%=-1.099$ in log odds space); for Picture Naming, Pinyin measure, there is no chance

919 and we therefore took minimal performance as making one correct response in the test ${ }^{6}$ (i.e. $1 / 72$

$920=-4.263$ in log odds space). For the cases where we are estimating an interaction between test-

921 session and variability-condition we set $x$ as equal to the mean increase in performance from pre-

922 and post- test across conditions (main effect of test-session). The logic is as follows: the

923 maximum difference is seen if low variability participants show no effect of test-session (no

924 improvement) and high variability participants show a positive effect of test-session. In this case,

925 if the mean effect of test-session is $\bar{t}$, the difference in $t$ between the two conditions will be equal

926 to $2 \bar{t}$. Again, we can set our estimate of $x$ to be half this value (i.e. $x=\bar{t}$ ).

927 We interpret BFs using the following conventions: $B<1 / 3$ indicates substantial evidence

928 for the null, $B>3$ indicates substantial evidence for $\mathrm{H} 1$, values between $1 / 3$ and 3 indicate that

929 the data collected do not sensitively distinguish H0 from H1 (Jeffreys 1961; Dienes 2008). Since

930 there is subjectivity in how the values for $\mathrm{H} 1$ are determined, we indicate the robustness of

931 Bayesian conclusions by reporting a robustness region for each $B$, which gives the range of

932 values of the scale factor $x$ that qualitatively support the same conclusion (i.e. evidence as

${ }^{6}$ Note that we cannot compute log-odds of 0. 
933 supporting $\mathrm{H} 0$, or as supporting $\mathrm{H} 1$, or there not being much evidence at all). Note that for

934 evidence for H0, the maximum $x$ is always infinity. The results are reported in Table 5 . It can be

935 seen we have substantial or strong evidence for the null for every test except for the Word

936 Repetition test for the Pinyin accuracy measure, where the evidence is ambiguous, and that the

937 robustness regions indicate that we would continue to have evidence for the null even with

938 smaller estimates of the scale factor $x$.

939

3.7.2 H1: There is an interaction between an individual's tone-aptitude and variabilitycondition, such that participants with greater tone-aptitude show greater performance following the multiple speaker conditions (HV and HVB) and those with lesser tone aptitude show greater performance in the single speaker condition (LV) We aimed to compute Bayes Factors comparing this hypothesis to the null for each of our data sets. We take the same approach as above except that we also compute Bayes factors for Training data, and for the Picture Identification test we look at both trained voice and untrained voice data - pooling the two in order to maximize available evidence. This is because this interaction has been reported with trained items (Sadakata \& McQueen, 2013) as well as untrained items (Perrachione et al., 2011). We again combine the HV and HVB conditions except for training where we look at the LV versus HV and LV versus HVB contrasts separately, 950 since we have seen in our previous analyses that HV and HVB are quite different (HVB 951 participants show higher performance).We again combine the evidence from trained and untrained items in the pre-post tests. For the post-session only tests, we are interested in the evidence for an interaction between the variability-condition contrast and aptitude. For the tests which appeared both pre- and post- training, we are interested in the interaction between the variability-condition contrast, aptitude and test-session. For training we look at the evidence for 
957 containing the interaction with training-session did not converge). As in our frequentist analyses

958 of aptitude, for the production measures - Word Repetition and Picture Naming - we do not look

959 at the pinyin measures since our aptitude measure is relevant only to tone learning.

960 We computed Bayes factors following the same procedure as in Section 3.7.1 and again

961 derived our estimates of the scale factor $x$ - the difference predicted under H1 - using the scale

962 and/or values from elsewhere in the data. Specifically, for each of the cases where we predicted a

963 two-way interaction between variability-condition and aptitude we set $x$ as equal to the mean

964 effect of aptitude across conditions (main effect of aptitude) ${ }^{7}$. The logic is as follows: The

965 maximum difference is seen if low variability participants show no effect of aptitude and the high

966 variability participants show a positive effect of aptitude (note that a negative effect of aptitude

967 is not expected in any condition). In this case, if the mean effect of aptitude is $\bar{a}$, the difference in

$968 a$ between the two conditions will be equal to $2 \bar{a}$. Again, we can set our estimate of $x-$ the $S D$ of

969 the half normal - to be half this maximum value i.e. $x=\bar{a}$. For the cases where we are interested

970 in the three-way interaction between aptitude, test-condition and test-session, we based our

971 estimate on half the difference between the maximal effect of aptitude (maxA - taken from the

972 scale) and their actual aptitude score at pre-test (baseline A - taken from the data). The logic is as

973 follows: The maximal effect of the interaction would be seen if participants in the low variability

974 condition showed the same baseline effect of aptitude at pre-test and at post-test ( $b a$ ), whereas

975 participants in the high variability condition showed maximal improvement at post-test (maxa).

976 In this case, the interaction between aptitude and session for the high variability group would be

\footnotetext{
${ }^{7}$ An alternative which would be more equivalent to the other BF analyses would be to inform the effect using the value of the two-way interaction of aptitude: test-session. We do not do this since we did not find an effect of this two-way interaction in either data set.
} 
977 equal to: maxa-ba. Again, we can set our estimate of $x$ - the $S D$ of the half normal - to be half

978 this maximum value, i.e. $x=\frac{m a-b a}{2}$

979

The maximum effect of aptitude was computed from the scale and the length of the

980 aptitude predictor. Specifically, we assumed that the maximal effect of aptitude would be

981 obtained if participants with maximal aptitude were at ceiling (71/72 correct $-\log$ odds 4.263$)$

982 and those with minimal aptitude were at chance (25\% in Word Repetition, Tone Accuracy, log

983 odds $=1.099 ; 33.33 \%$ in Three Interval Oddity, $\log$ odds $=0.693$ ). We divided this range by the

984 length of the aptitude predictor to obtain a measure of a one-step change in aptitude.

985 The results are summarised in Table 6. It can be seen that although there is more evidence for the

986 null than $\mathrm{H} 1$ in each case (i.e. BF $<1$ ) we do not have substantial evidence for the null over H1

987 in any case. Thus, we cannot draw any inferences about the interaction from this data. Note that,

988 in most cases, the robustness regions indicate that even if the scale factor $x$ was twice as large,

989 i.e. corresponding to the maximum value we might expect, the $B$ would be ambiguous.

9904 Discussion

991 The current study investigated the effect of different types of phonetic training on English

992 speakers' learning of novel Mandarin words and tones. To our knowledge, this is the first study

993 to train naive participants on all four Mandarin tones, using real language stimuli embedded in a

994 word learning task. Learning was examined using a range of perception and production tasks.

995 Following previous literature, we compared three training conditions: low variability (single

996 speaker), high variability (four speakers, presented intermixed) and high variability blocked (four

997 speakers, presented in blocks). We also administered tests designed to tap individual aptitude in

998 the perception of pitch contrasts, adapted from the previous literature. The results indicated that

999 participants' performance increased during training and that training also led to improved 
1000 performance on pre- to post- tests of discrimination and production, with evidence of

1001 generalisation to untrained voices and items. Participants also showed some ability to recall

1002 trained words - including their tones - in a picture naming task administered at post-test.

1003 However, the only place where we saw any effect of the variability manipulation was in the

1004 training task (and with trained items in the picture identification task, which was highly similar

1005 to training), where the low variability group outperformed both of the high variability groups.

1006 Critically, we found no evidence in any of our tests that high variability input benefitted learning

1007 or generalisation, nor did we find any evidence of an interaction between individual aptitude and

1008 the ability to benefit from high variability training. In the following discussion, we first consider

1009 the findings from each task in turn before turning to a more general discussion of our findings in

1010 relation to the predicted benefit of high variability input.

\section{$10114.1 \quad$ Tests of individual aptitude}

1012 In the current work, we conducted two tests with the purpose of capturing individual

1013 aptitude: The Pitch Contrast Perception Test (following Perrachione et al 2011) and the

1014 Categorisation of Synthesised Tonal Continua, following Sadakata and McQueen (2014).

1015 Although our goal was to measure participants' baseline aptitude, the tests were conducted both

1016 at pre- and post- test, following Sadakata and McQueen, who did not find differences from pre-

1017 to post- tests with their categorisation measure, and who used combined data from pre- and post-

1018 test to compute participants slopes. Unfortunately, the performance of our own participants

1019 suggested that the Categorisation of Synthesised Tonal Continua test was not a good test of

1020 aptitude, with the majority of participants failing to meet the slope threshold used in Sadakata

1021 and McQueen, and most being unable to consistently categorise the end points of the continua. It

1022 is unclear why our results differ from the previous study (we aimed to follow their procedures), 
1023 but this meant that we were unable to use this as an aptitude measure in our later analyses. The

1024 scores on the Pitch Contrast Perception Test alone therefore served as our measure of individual

1025 aptitude. Interestingly, preliminary analyses (Section 3.2.1) demonstrated that performance in

1026 this test improved from pre- to post- training. This suggests that this measure is not a "pure"

1027 measure of individual differences since it also appears to be affected by experience. Given this,

1028 we only used participants' scores on this test from pre-test as the measure of aptitude in

1029 subsequent analyses.

\section{$1030 \quad 4.2 \quad$ Performance in Training}

1031 The training task employed in this study was a 2AFC task, where participants had to

1032 identify the correct meaning of a Mandarin word based on its tone. The results from training

1033 indicate that participants performed better in the single speaker LV training than in either the

1034 multiple speaker HV or HVB groups. This difference was present from the first session for the

1035 LV-HV contrast, and from the second session for the LV-HVB contrast (i.e. the first session

1036 where the two conditions differ), and increased over time for both contrasts. Greater difficulty

1037 with multiple speaker input is in line with the findings of Perrachione et al. (2011), although the

1038 differences did not emerge so rapidly in that study, possibly due to there being fewer trials per

1039 session. Intuitively, repeated exposure to the single speaker in the LV condition allows for

1040 greater adaptation to speaker specific cues, whereas in the HV conditions participants have to

1041 adapt to multiple speakers. This is particularly difficult in the unblocked HV condition, where

1042 trial-by-trial adaptation is needed, which is effortful for participants (Magnuson \& Nusbaum,

1043 2007). Importantly, however, for all three groups, their performance gradually increased over

1044 each session. In combination with the fact that their performance on the other tasks increased

1045 after training, this indicates that the training task and materials were effective. We also explored 
1046 the role of learner aptitude in this task (as measured by performance on the Pitch Contour

1047 Perception Test at pre-test) and whether this influenced participant's performance differently in

1048 the different variability conditions. Overall, aptitude was found to be a significant predictor of

1049 performance during training. However, there was no evidence for an interaction with training

1050 condition, although our Bayes Factor Analyses suggests that the data here are inconclusive. We

1051 return to this finding in Section 4.5 below.

\section{$1052 \quad 4.3 \quad$ Perception Tests}

1053 We included two perceptual tasks which tapped learning and generalisation due to

1054 training: A Picture Identification administered at post-test and a Three Interval Oddity task

1055 administered at both pre- and post-test. The Picture Identification task was a version of the

1056 training task without feedback, and is the most similar to the tests used by Perrachione et al.

1057 (2011), and Sadakata and McQueen (2014). We used this test to look at learning of the trained

1058 stimuli, comparing trained and untrained voices. The three interval oddity task had not been used

1059 in the previous studies, but allowed us to use a pre- /post- test design, and also to look at

1060 participants' performance with untrained items. These tests provided evidence that participants

1061 improved in their perception of tones following training: They were above chance in using the

1062 tone to identifying the correct picture in the picture identification task at post-test, and they

1063 improved in their ability to discriminate tones in the three interval oddity task (59\% performance

1064 prior to training, $66 \%$ post training). There was also evidence of generalisation across both

1065 voices and items: Participants were above chance in identifying the correct pictures even with an

1066 untrained voice (although they did show significantly weaker performance than with the trained

1067 voiced) and they improved in their ability to discriminate the between minimal pair items in the

1068 three interval oddity task, even for untrained items. 
1069 Our key questions concerned the role of variability in training. First, we were interested in

1070 whether there was evidence that exposure to multiple voices during training led to greater ability

1071 to generalise across voices at test - i.e. greater performance with novel in the high variability

1072 conditions than in the low variability condition. We did not see this. In fact, the only effect of

1073 variability in this data was a low variability benefit, which we saw in the Picture Identification

1074 task for the trained-voice items (seen in the contrast between LV and HV condition). This

1075 mirrors what we saw in training and reflects the greater exposure to this particular speaker in the

1076 low variability training. However, in the tests tapping generalisation to a novel speaker - i.e. in

1077 untrained voice trials in the Picture Identification task, and with all of the test-items in the Three

1078 Interval Oddity task, there was no difference between variability-training conditions. Bayes

1079 factor analyses indicate that in both cases, there was substantial evidence for the null.

1080 The second hypothesis was that there would be an interaction between learner aptitude

1081 (as measured by the Pitch Contour Perception Test at pre-test) and variability training condition,

1082 such that high aptitude participants would benefit more from high variability training. Note that

1083 previous work had found this interaction both in tests involving generalisation (Perrachione et

1084 al., 2011) and with trained items (Sadakata \& McQueen, 2014) so we considered both in our

1085 analyses here. There was no evidence of such an interaction in either the Picture Identification or

1086 Three Interval Oddity tasks. However, Bayes Factor analyses suggest that the data are

1087 inconclusive. We return to these points in Section 4.5 below.

1088 Another finding from the Three Interval oddity test that is worth noting, although it did

1089 not concern our hypotheses, is that some trial types were harder than others. Recall that this test

1090 involved participants hearing three different stimuli each produced by a different speaker, which

1091 makes noting the similarity across two of the stimuli much harder - something we discovered in 
1092 pilot work, where even before training participants were near ceiling with an equivalent task in

1093 which the same speaker produced all three stimuli within a single trial. However, analyses of

1094 trial-type demonstrated that participants were additionally affected by the gender of the three

1095 speakers producing each of the stimuli. Specifically, at pre-test, participants showed best

1096 performance for trials where one of the speakers was male and the other two were female, and

1097 the target "odd man" was the male speaker ("easy" trials). In contrast, they showed worst

1098 performance if there was one male and two female speakers, but the "odd man" was one of the

1099 female speakers ("hard" trials). Middle level performance was shown for trials where all three

1100 speakers were female ("neutral" trials). This is presumably due to participants relying on

1101 perceptual cues associated with speaker gender to do the task. Interestingly, our analyses showed

1102 that performance only increased for the trials where the odd one was not the lone male (the

1103 "neutral" and "hard" ones), but not for those where the male was the odd man. Given that

1104 participants are not near ceiling at pre-test (67\%), it is perhaps surprising that their trained

1105 knowledge of the tone contrasts does not boost their performance. One possibility is although

1106 they are now better able to use tone cues, they are also less likely to use gender based cues,

1107 which they may now realize are less reliable, masking improvement based on tone for these

1108 particular test items.

1109 4.4 Production Tasks

1110 In this study, we used two production tasks, a word repetition task administered pre and

1111 post training, in which participants repeated back Mandarin words, and a Picture Naming task

1112 testing vocabulary recall, which was administered at post-test only. High variability perceptual

1113 training for tones has been previously found to transfer to production (Bradlow and Pisoni, 1999; 
1114 Zeromskaite, 2014), however the benefits of high variability and low variability training have not

1115 been contrasted.

1116 In the Word Repetition task, there was a significant, though relatively modest

1117 improvement in participants' ability to reproduce the tone of the stimuli, such that it could be

1118 identified by a native speaker (from pre- to post- test: $70 \%$ to $76 \%$ ) and in the Picture Naming

1119 task, participants showed an ability to recall and reproduce the correct tone, although

1120 unsurprisingly with less accuracy than in the repetition task (50\%). For Word Repetition, we

1121 were also able to look at transfer to untrained words: As in the perception tasks, there was once

1122 again equivalent improvement for both trained and untrained items. Together, these results

1123 provide evidence that purely perceptual training on tone contrast can transfer to production, as

1124 well as to novel items.

1125 In addition to looking at the production of tones, we also looked at participants' ability to

1126 produce the correct segmental phonology (pinyin-score). Participants showed a small but

1127 significant improvement on this measure in Word Repetition (54\% correct at pre-test, $58 \%$ at

1128 post-test), and some ability to recall the segments in the Picture Naming test (50\% correct). This

1129 indicates some learning of segmental phonology due to training, despite the fact that the focus of

1130 the training task was on training tonal information through the presentation of tonal minimal-

1131 pairs.

1132 Turning to the role of variability, the predicted benefit of high variability training was not

1133 evident in any of the measures in either of the production tasks, with Bayes factor analyses

1134 indicating substantial evidence for the null except for the Word Repetition pinyin-measure,

1135 where the evidence was ambiguous. With regard to aptitude, although performance on the Pitch

1136 Contour Perception Test at pre-test was predictive of participants' ability to produce tones in 
1137 both tasks (indicating a relationship between participants perceptual and production ability), we

1138 did not find the predicted interaction between aptitude and variability condition in either task.

1139 Here however, Bayes Factor analyses suggests that the results are inconclusive. We return to

1140 these points about variability below.

$11414.5 \quad$ The Role of High Variability Materials in Training and Generalisation

1142 In the current study, across all of the different tests, we did not find either an overall

1143 benefit of exposure to high variability training materials for generalisation, or any interaction

1144 between such a benefit and individual aptitude.

1145 We consider first the lack of overall variability benefit for generalisation. Importantly, in

1146 addition to finding a pattern of null results (i.e. $\mathrm{p}<.05$ ) in the frequentist analyses, additional

1147 Bayes Factor analyses also found substantial evidence for the null $(\mathrm{BF}<.33)$ in all but one of the

1148 test measures (Word Repetition, Pinyin, where BF $=.421$ ). Thus, there is good evidence that, at

1149 least for these training and test materials, exposure to stimuli from multiple speakers does not

1150 lead to greater generalisation in either perception or production. This finding is consistent with

1151 the lack of a main effect of variability condition in the transfer tasks in either Sadakata \&

1152 McQueen (2014) or Perrachione et al. (2011) (see also footnote 1). However it is at odds with

1153 other phonetic training studies focused on segmental contrasts (Clopper \& Pisoni, 2004; Logan et

1154 al. 1991, Lively et al., 1993; Sadakata \& McQueen 2013) and with the literature demonstrating a

1155 high variability benefit in vocabulary learning (Barcroft \& Sommers, 2005, 2014; Sommers \&

1156 Barcroft, 2007, 2011). This suggests that this overall variability benefit may be restricted to

1157 segmental rather than tonal phonetic learning, at least for speakers of a non-tonal L1.

1158 It is difficult to reconcile the lack of benefit for vocabulary learning in the picture naming

1159 task, given the findings of Barcroft, Sommers and colleagues $(2005,2007,2011,2014)$, since 
1160 this test is quite similar to that used in their experiments. However, one possibility is that this is

1161 due to differences in our training set up (i.e. focused on training tonal contrasts) compared with

1162 the earlier vocabulary studies. Nonetheless it remains unclear why tone learning should be

1163 different from other types of phonetic learning in terms of benefiting from talker-variability.

1164 Theoretically speaking, in a framework where all cues compete, variation in idiosyncratic

1165 speaker-specific cues would be expected to provide key evidence as to which cues are irrelevant

1166 to the phonetic contrast in question (Apfelbaum \& McMurray, 2011; Ramscar \& Baayen, 2013;

1167 Ramscar, Yarlett, Dye, Denny \& Thorpe, 2010). This raises the question of how participants in

1168 our low variability condition are able to generalize at all - i.e. how can they identify the

1169 phonetically relevant cues compared with the idiosyncratic cues associated with the single

1170 speaker to which they were exposed? One possibility is that other variation in our materials aided

1171 generalisation, in particular in our real word stimuli, each tone-contrast is encountered with

1172 multiple consonants and vowels. If item variability also aids generalisation to new speakers, this

1173 might explain why we found equivalent generalisation across conditions instead of seeing greater

1174 generalisation in the HV conditions (i.e. even the LV condition is really a high variability

1175 condition, because of the item variability). On the other hand, Sadakata and McQueen (2014)

1176 also saw generalisation even for their low variability condition, and in their study this condition

1177 lacked variation in terms of both speakers and phonetic contexts. This suggests that the relevant

1178 cues for the tone contrasts may be sufficiently acoustically salient for learners to identify them,

1179 even when exposure occurs in limited contexts.

1180 Another possibility - and the one suggested by the findings of Sadakata and McQueen

1181 (2014) and Perrachione et al. (2011) - is that benefits of high variability for generalisation are

1182 masked by individual differences. In their studies, only high aptitude participants showed a high 
1183 variability benefit, while low aptitude participants did not. It is possible that for lower aptitude

1184 participants, the benefits of exposure to varying, idiosyncratic cues are offset by the greater

1185 difficulty that these participants have in attuning to the different speakers during training, as

1186 discussed above (Section 4.1). This explanation is supported by evidence from a study by

1187 Goldinger, Pisoni and Logan (1991) who explored the effect of increasing the processing cost of

1188 multi-speaker input in the context of word recall (in the L1). Specifically, they exposed

1189 participants to single versus multi-speaker word lists, manipulating presentations rates. They

1190 found that single-speaker lists produced better word recall than multiple-speaker lists at short

1191 inter-word intervals (less than $2000 \mathrm{~ms}$ ) whereas this effect was reversed for longer inter-word

1192 intervals. This suggests that increasing encoding difficulty can remove the benefits of multi-

1193 speaker exposure. Relatedly, Sinkeviciute, Brown, Brekelmans, \& Wonnacott (in press; preprint)

1194 found that young learners have greater difficulty processing multi-speaker training materials in

1195 L2 vocabulary learning, and subsequently fail to show a speaker-variability benefit at test. One

1196 interpretation of these findings is that age-related capacity limitations may constrain the ability to

1197 benefit from speaker variability, supporting the notion that differences in capacity limitations can

1198 affect an individual's ability to benefit from multi-talker training.

1199 Returning to the current study, we did not find an interaction between variability-training

1200 and learner aptitude. However, it is important to acknowledge the results of our Bayes factor

1201 analyses, which did not find substantial evidence in support of the null over H1 (or H1 over H0)

1202 for any of the test tasks. This means that we cannot draw conclusions about this hypothesis from

1203 the current data. In theory, we could continue collecting data until we had substantial evidence

1204 for either $\mathrm{H} 0$ or $\mathrm{H} 1$. To explore the feasibility of this, we conducted supplementary analyses to

1205 estimate the sample size that might be needed to see substantial evidence for the null (based on 
1206 the assumption that the error term would reduce in proportion to $\sqrt{\mathrm{SE}}$ ). Taking the Picture

1207 Identification test (the test most similar to previous studies) our results suggests that it would

1208 require $\mathrm{N}>300-$ i.e. over five times our current sample size. This suggests that this

1209 experimental paradigm is not sufficiently sensitive to address this hypothesis.

1210 Given the ambiguity of our findings with regard to the interaction, it is not appropriate to

1211 extensively interpret why we do not find the interaction while the previous studies did. However,

1212 we note that there are a variety of differences across the studies which could underpin the

1213 different findings, if it holds true. For example, the test of individual differences which we use is

1214 harder than that used by Perrachione et al. (2011) since it uses all six Mandarin vowels (whereas

1215 the original study used five, without / $\mathrm{u} /$ ) and all of the Mandarin tones (where Perrachione et al.

1216 used three, without Tone 3). This change also means that that we cannot easily contrast the range

1217 of participant scores in the two studies and it may be that the spread of ability of our participant

1218 is different from theirs. In addition, our training task is potentially harder than both of the

1219 previous studies, i.e. involving all four tones in the context of natural Mandarin stimuli in the

1220 context of a word learning tasks. Finally, we also note that our statistical analyses are different

1221 from both of the previous studies in that they took their continuous aptitude measures and turned

1222 these into binary factors using a "cut off", whereas our statistical approach allows us to use them

1223 as continuous variables. However, this should in principle make our approach more powerful

1224 than in previous studies.

\section{$1225 \quad 4.6 \quad$ Future Directions}

1226 If the interaction between aptitude and training condition reported in Sadakata and

1227 McQueen (2014) and Perrachione et al., (2011) is to have implications for educational materials,

1228 it is important to establish whether it extends to other more naturalistic materials. Given the 
1229 relatively small samples in these original studies, and the increasing recognition that psychology

1230 experiments have been routinely underpowered (Maxwell, Lau \& Howard, 2015; and see

1231 Vasishth, Mertzen, Jäger, \& Gelman, (2018) for a recent demonstration in the area of reading)

1232 and that can lead to increases in both type 1 and type 2 error, we suggest that it would be useful

1233 to implement a direct, high powered replication of these previous studies. We note that having a

1234 sufficient sample to provide substantial evidence for $\mathrm{H} 1 / \mathrm{H} 0$ using Bayesian methods, or to obtain

$123590 \%$ power for frequentist methods, would likely require a much larger sample than is standard

1236 in these types of studies. Given the time-consuming nature of these multiple session training

1237 studies, moving to online testing may be necessary to make this feasible (see Xie et al. 2018 for

1238 an example of an acoustic training study done over the web), or alternately multi-lab

1239 collaboration may be necessary. Note that this would also allow us to see whether the fact that

1240 Perrachione et al., (2011) found their interaction with untrained voices, whereas Sadakata \&

1241 McQueen (2014) found it only for trained voices, is a true difference (due to the different

1242 paradigms) or due to power. Critically, successful replication would allow us to then extend the

1243 paradigms in such a way as to explore the factors above. For example, would increasing the

1244 number of tones to use all four Mandarin tones and/or using natural Mandarin stimuli affect the

1245 interaction between variability in the input and learner aptitude?

1246 Although direct replication will play a useful role in establishing these effects, we believe

1247 that ultimately it will also be important to develop a more nuanced approach to measuring the

1248 factors leading to different levels of aptitude both in tone learning, and in other types of phonetic

1249 learning. We note that here in addition to not seeing the predicted interaction with variability, we

1250 also didn't see interactions between aptitude and training session in any of our tasks, suggesting

1251 that our aptitude measure predicted baseline performance on the task and not the ability to 
1252 improve due to training. In addition, the tasks used to measure "aptitude" are quite similar in

1253 nature to the training and test tasks, decreasing their explanatory value. Our ongoing work

1254 explores the combined predictive value of a range of measures including measures of attention,

1255 working memory and musical ability. Identifying factors which are predictive of aptitude for

1256 tone learning has clear implications for teaching and the personalisation of teaching methods.

\section{Conclusion}

1258 We trained naive participants on all four Mandarin tones, using real language stimuli

1259 embedded in a word learning task. We found improvements in both production and perception of

1260 tones which transferred to novel voices and items. We found that learning was greatest for

1261 training with a single voice but that training with a single voice versus four voices (whether

1262 intermixed or blocked) lead to equal amounts of generalisation. Although learner aptitude

1263 predicted performance in most tasks, there was no evidence that different levels of aptitude lead

1264 to better or worse learning from different types of training input. 


\section{References}

1266 Aliaga-García, C., \& Mora, J. C. (2009). Assessing the effects of phonetic training on L2 sound

1267 perception and production. In M. A. Watkins, A. S. Rauber, \& B.O. Baptista (Eds.). Recent

1268 Research in Second Language Phonetics/Phonology: Perception and Production (pp. 2-

1269 31). Newcastle upon Tyne, UK: Cambridge Scholars Publishing.

1270 Audacity Team. (2015). Audacity (Version 2.1.1). Computer Program. Retrieved May, 2015, 1271 from $\underline{\text { http://audacityteam.org/ }}$

1272 Alshangiti, W., \& Evans, B. G. (2014, May). Investigating the domain-specificity of phonetic

1273 training for second language learning: Comparing the effects of production and perception

1274 training on the acquisition of English vowels by Arabic learners of English. In the

1275 Proceedings of the International Seminar for Speech Production, Cologne, Germany.

1276 Apfelbaum, K. S., \& McMurray, B. (2011). Using variability to guide dimensional weighting:

1277 Associative mechanisms in early word learning. Cognitive Science, 35(6), 1105-1138.

1278 https://doi.org/10.1111/j.1551-6709.2011.01181.x

1279 Baayen, R. H., Davidson, D. J., \& Bates, D. M. (2008). Mixed-effects modeling with crossed

$1280 \quad$ random effects for subjects and items. Journal of Memory and Language, 59(4), 390-412.

$1281 \quad$ https://doi.org/10.1016/j.jml.2007.12.005

1282 Barcroft, J., \& Sommers, M. S. (2005). Effects of acoustic variability on second language

1283 vocabulary learning. Studies in Second Language Acquisition, 27, 387-414.

$1284 \quad$ https://doi.org/10.1017/S0272263105050175

1285 Barcroft, J., \& Sommers, M. S. (2014). Effects of variability in fundamental frequency on L2

1286 vocabulary learning: A comparison between learners who do and do not speak a tone 
1287

1288

1289

1290

1291

1292

1293

1294

1295

1296

1297

1298

1299

1300

1301

1302

1303

1304

1305

1306

1307

1308

1309

language. Studies in Second Language Acquisition, 36(3), 423-449. $\underline{\text { https://doi.org/10.1016/j.neuropsychologia.2006.11.015 }}$

Barr, D. J., Levy, R., Scheepers, C., \& Tily, H. J. (2013). Random effects structure for confirmatory hypothesis testing: Keep it maximal. Journal of Memory and Language, 68(3), 255-278. https://doi.org/10.1016/j.jml.2012.11.001

Bates, D., Maechler, M., Bolker, B., \& Walker, S. (2013). lme4: Linear mixed-effects models using Eigen and S4. R package version 1.0-5. 2013.

Boersma, P., \& Weenink, D. (2015). Praat: doing phonetics by computer [Computer program]. Version 5.4.14, retrieved 24 July 2015 from http://www.praat.org/

Bradlow, A. R., \& Bent, T. (2008). Perceptual adaptation to non-native speech. Cognition, 106(2), 707-729. https://doi.org/10.1016/j.cognition.2007.04.005

Bradlow, A. R., \& Pisoni, D. B. (1999). Recognition of spoken words by native and non-native listeners: Talker-, listener-, and item-related factors. The Journal of the Acoustical Society of America, 106, 2074-2085. http://dx.doi.org/10.1121/1.427952

Bradlow, A. R., Akahane-Yamada, R., Pisoni, D. B., \& Tohkura, Y. I. (1999). Training Japanese listeners to identify English/r/and/l: Long-term retention of learning in perception and production. Perception \& Psychophysics, 61(5), 977-985. https://doi.org/10.3758/BF03206911.

Bygate, M., Swain, M., \& Skehan, P. (2013). Researching pedagogic tasks: Second language learning, teaching, and testing. London UK: Routledge.

Cicchetti, D. V. (1994). Guidelines, criteria, and rules of thumb for evaluating normed and standardized assessment instruments in psychology. Psychological Assessment, 6(4), 284. http://dx.doi.org/10.1037/1040-3590.6.4.284 
1310 Clopper, C. G., \& Pisoni, D. B. (2004). Some acoustic cues for the perceptual categorization of

1311 American English regional dialects. Journal of Phonetics, 32(1), 111-140.

1312 https://doi.org/10.1016/S0095-4470(03)00009-3

1313 Cohen, A. D. (2014). Strategies in learning and using a second language. London UK:

$1314 \quad$ Routledge.

1315 Dienes, Z. (2008). Understanding psychology as a science: An introduction to scientific and

1316 statistical inference. Macmillan International Higher Education.

1317 Dienes, Z. (2014). Using Bayes to get the most out of non-significant results. Frontiers in

1318 psychology, 5, 781. https://doi.org/10.3389/fpsyg.2014.00781

1319 Dienes, Z. (2015). How Bayesian statistics are needed to determine whether mental states are

1320 unconscious. In M. Overgaard (Ed.), Behavioural methods in consciousness research (pp.

1321 199-220). Oxford: Oxford University Press.

1322 Dienes, Z., Coulton, S., \& Heather, N. (2018). Using Bayes factors to evaluate evidence for no

1323 effect: examples from the SIPS project. Addiction, 113(2), 240-246.

$1324 \quad$ https://doi.org/10.1111/add.14002

1325 Flege, J. E., Takagi, N., \& Mann, V. (1995). Japanese adults can learn to produce

1326 English/I/and/1/accurately. Language and Speech, 38, 25-55.

1327 https://doi.org/10.1177/002383099503800102

1328 Giannakopoulou, A., Brown, H., Clayards, M., \& Wonnacott, E. (2017). High or low?

1329 Comparing high and low variability phonetic training in adult and child second language

1330 learners. PeerJ, 5, e3209. DOI:10.7717/peerj.3209

1331 Giannakopoulou, A., Uther, M., \& Ylinen, S. (2013). Enhanced plasticity in spoken language

1332 acquisition for child learners: Evidence from phonetic training studies in child and adult 
learners of English. Child Language Teaching and Therapy, 29, 201-218. https://doi.org/10.1177/0265659012467473

1335 Hay, J., Drager, K., \& Thomas, B. (2013). Using nonsense words to investigate vowel

1336 merger. English Language \& Linguistics, 17(2), 241-269.

1337 Iverson, P., Ekanayake, D., Hamann, S., Sennema, A., \& Evans, B. G. (2008). Category and

1338 perceptual interference in second-language phoneme learning: An examination of

1339 English/w/-/v/learning by Sinhala, German, and Dutch speakers. Journal of Experimental

1340 Psychology: Human Perception and Performance, 34, 1305. https://doi.org/10.1037/0096-

$1341 \quad \underline{1523.34 .5 .1305}$

1342 Iverson, P., Hazan, V., \& Bannister, K. (2005). Phonetic training with acoustic cue

1343 manipulations: A comparison of methods for teaching English /r/-/1/ to Japanese

1344 adults. The Journal of the Acoustical Society of America, 118, 3267-3278.

$1345 \quad$ https://doi.org/10.1121/1.2062307

1346 Jaeger, T. F. (2008). Categorical data analysis: Away from ANOVAs (transformation or not) and

1347 towards logit mixed models. Journal of Memory and Language, 59(4), 434-446.

$1348 \quad \underline{\text { https://doi.org/10.1016/j.jml.2007.11.007 }}$

1349 Jeffreys, H. (1998). The theory of probability. OUP Oxford.

1350 Joanisse, M. F., Manis, F. R., Keating, P., \& Seidenberg, M. S. (2000). Language deficits in

1351 dyslexic children: Speech perception, phonology, and morphology. Journal of

1352 Experimental Child Psychology, 77, 30-60. https://doi.org/10.1006/jecp.1999.2553

1353 Lambacher, S. G., Martens, W. L., Kakehi, K., Marasinghe, C. A., \& Molholt, G. (2005). The

1354 effects of identification training on the identification and production of American English 
vowels by native speakers of Japanese. Applied Psycholinguistics, 26(2), 227-247. https://doi.org/10.1017/S0142716405050150

1357 Landis, J. R., \& Koch, G. G. (1977). The measurement of observer agreement for categorical 1358 data. Biometrics, 33(1),159-174. DOI: $10.2307 / 2529310$

1359 Lengeris, A., \& Hazan, V. (2010). The effect of native vowel processing ability and frequency

1360 discrimination acuity on the phonetic training of English vowels for native speakers of

1361 Greek. The Journal of the Acoustical Society of America, 128, 3757-3768.

$1362 \quad$ https://doi.org/10.1121/1.3506351

1363 Lively, S. E., Logan, J. S., \& Pisoni, D. B. (1993). Training Japanese listeners to identify

1364 English/r/and/1/. II: The role of phonetic environment and talker variability in learning new

1365 perceptual categories. The Journal of the Acoustical Society of America, 94, 1242-1255.

1366 https://doi.org/10.1121/1.408177

1367 Logan, J. S., Lively, S. E., \& Pisoni, D. B. (1991). Training Japanese listeners to identify

1368 English/r/and/1/: A first report. The Journal of the Acoustical Society of America, 89, 874-

1369 886. https://doi.org/10.1121/1.1894649

1370 Magnuson, J. S., \& Nusbaum, H. C. (2007). Acoustic differences, listener expectations, and the

1371 perceptual accommodation of talker variability. Journal of Experimental Psychology:

1372 Human Perception and Performance, 33, 391. https://doi.org/10.1037/0096-1523.33.2.391

1373 Mattys, S. L., \& Wiget, L. (2011). Effects of cognitive load on speech recognition. Journal of

1374 Memory and Language, 65(2), 145-160. https://doi.org/10.1016/j.jml.2011.04.004

1375 Maxwell, O., Baker, B., Bundgaard-Nielsen, R., \& Fletcher, J. (2015). A comparison of the

1376 acoustics of nonsense and real word stimuli: Coronal stops in Bengali. Proceedings of the

1377 meeting of the International Congress of Phonetic Sciences, Glasgow, UK. 
1378 Maxwell, S. E., Lau, M. Y., \& Howard, G. S. (2015). Is psychology suffering from a replication

1379 crisis? What does "failure to replicate" really mean? American Psychologist, 70(6), 487.

$1380 \quad$ http://dx.doi.org/10.1037/a0039400

1381 McGraw, K. O., \& Wong, S. P. (1996). Forming inferences about some intraclass correlation

1382 coefficients. Psychological Methods, 1(1), 30. http://dx.doi.org/10.1037/1082-989X.1.1.30

1383 Nusbaum, H. C., \& Morin, T. M. (1992). Paying attention to differences among talkers. Speech

1384 perception, production and linguistic structure, (pp. 113-134). Amsterdam, Netherlands:

$1385 \quad$ IOS press.

1386 Perrachione, T. K., Lee, J., Ha, L. Y., \& Wong, P. C. (2011). Learning a novel phonological

1387 contrast depends on interactions between individual differences and training paradigm

1388 design. The Journal of the Acoustical Society of America, 130, 461-472.

1389 https://doi.org/10.1371/journal.pone.0089642

1390 Powell, M. J. (2009). The BOBYQA algorithm for bound constrained optimization without

1391 derivatives. Cambridge NA Report NA2009/06, University of Cambridge, Cambridge, 26-

139246.

1393 Quené, H., \& Van den Bergh, H. (2008). Examples of mixed-effects modeling with crossed

1394 random effects and with binomial data. Journal of Memory and Language, 59(4), 413-425.

1395 https://doi.org/10.1016/j.jml.2008.02.002

1396 Ramscar, M., \& Baayen, H. (2013). Production, comprehension, and synthesis: a communicative

1397 perspective on language. Frontiers in psychology, 4, 233.

$1398 \quad$ https://doi.org/10.3389/fpsyg.2013.00233 
1399 Ramscar, M., Yarlett, D., Dye, M., Denny, K., \& Thorpe, K. (2010). The effects of

1400 feature-label-order and their implications for symbolic learning. Cognitive science, 34(6), 1401 909-957. https://doi.org/10.1111/j.1551-6709.2009.01092.x

1402 R Development Core Team (2010). R: A Language and Environment for Statistical Computing,

$1403 \quad$ Version R 3.3.2. Available at www. r-project. org. Accessed September 2017.

1404 Sadakata, M., \& McQueen, J. M. (2013). High stimulus variability in nonnative speech learning 1405 supports formation of abstract categories: Evidence from Japanese geminates. The Journal 1406 of the Acoustical Society of America, 134, 1324-1335. https://doi.org/10.1121/1.4812767

1407 Sadakata, M., \& McQueen, J. M. (2014). Individual aptitude in Mandarin lexical tone perception 1408 predicts effectiveness of high-variability training. Frontiers in Psychology, 5, 1318.

$1409 \quad$ https://doi.org/10.3389/fpsyg.2014.01318

1410 Scarborough, R. (2012). Lexical similarity and speech production: Neighborhoods for nonwords.

1411 Lingua, 122(2), 164-176. https://doi.org/10.1016/j.lingua.2011.06.006

1412 Sommers, M. S., \& Barcroft, J. (2007). An integrated account of the effects of acoustic

1413 variability in first language and second language: Evidence from amplitude, fundamental

1414 frequency, and speaking rate variability. Applied Psycholinguistics, 28(2), 231-249.

$1415 \quad$ https://doi.org/10.1017/S0142716407070129

1416 Sommers, M. S., \& Barcroft, J. (2011). Indexical information, encoding difficulty, and second

1417 language vocabulary learning. Applied Psycholinguistics, 32(2), 417-434.

1418 https://doi.org/10.1017/S0142716410000469

1419 Strange, W., \& Dittmann, S. (1984). Effects of discrimination training on the perception of $/ \mathrm{r} / \mathrm{-l} /$

1420 by Japanese adults learning English. Perception \& Psychophysics, 36, 131-145.

$1421 \quad \underline{\text { https://doi.org/10.3758/BF03202673 }}$ 
1422 Strange, W., \& Shafer, V. L. (2008). Speech perception in second language learners: The re-

1423 education of selective perception. Phonology and second language acquisition (pp.153-

1424 192). Amsterdam, Netherland: John Benjamins Publishing Company.

1425 Vasishth, S., Mertzen, D., Jäger, L. A., \& Gelman, A. (2018). The statistical significance filter

1426 leads to overoptimistic expectations of replicability. Journal of Memory and Language,

1427 103, 151-175. https://doi.org/10.1016/j.jml.2018.07.004

1428 Wang, Y., Jongman, A., \& Sereno, J. A. (2003). Acoustic and perceptual evaluation of Mandarin

1429 tone productions before and after perceptual training. The Journal of the Acoustical Society

1430 of America, 113, 1033-1043. https://doi.org/10.1121/1.1531176

1431 Wang, Y., Spence, M. M., Jongman, A., \& Sereno, J. A. (1999). Training American listeners to

1432 perceive Mandarin tones. The Journal of the Acoustical Society of America, 106, 3649-

1433 3658. https://doi.org/10.1121/1.428217

1434 Wong, J. (2014). The Effects of High and Low Variability Phonetic Training on the Perception

1435 and Production of English Vowels /e/-/æ/ by Cantonese ESL Learners with High and Low

1436 L2 Proficiency Levels. Proceedings of the 15th Annual Conference of the International

1437 Speech Communication Association, 524-528. Retrieved from

1438 https://repository.hkbu.edu.hk/hkbu_staff_publication/6234.

1439 Wong, P., \& Perrachione, T. K. (2007). Learning pitch patterns in lexical identification by native

$1440 \quad$ English-speaking adults. Applied Psycholinguistics, 28, 565-585.

$1441 \quad$ https://doi.org/10.1017/S0142716407070312

1442 Xie, X., Weatherholtz, K., Bainton, L., Rowe, E., Burchill, Z., Liu, L., \& Jaeger, T. F. (2018).

1443 Rapid adaptation to foreign-accented speech and its transfer to an unfamiliar speaker. The 
1444 Journal of the Acoustical Society of America, 143, 2013-2031.

$1445 \quad$ https://doi.org/10.1121/1.5027410

1446 Yip, M. (2002). Tone. Cambridge textbooks in linguistics. Cambridge: Cambridge University

$1447 \quad$ Press.

1448 Zeromskaite, I. (2014). The potential role of music in second language learning: A review

1449 article. Journal of European Psychology Students, 5, 78-88. http://doi.org/10.5334/jeps.ci 
Figure 1

Tasks completed in each of the eight sessions

This figure describes all tasks arranged through session 1-8

SESSION 1
1) Pitch Contour Perception
Test
2) Categorisation of
Synthesised Tonal Continua
3) Word Repetition
4) Three Interval Oddity
5) English Introduction

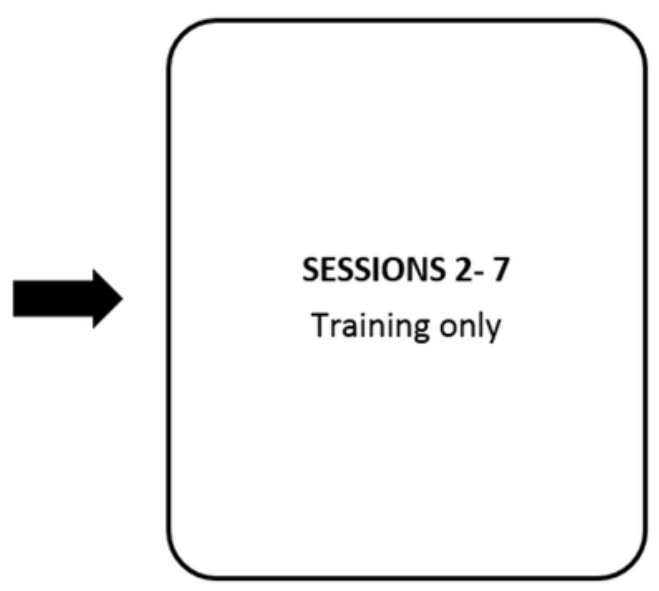

\begin{tabular}{|l|}
\multicolumn{1}{c}{ SESSION 8} \\
1) Word Repetition \\
2) Three Interval Oddity \\
3) Picture Identification \\
4) Pitch Contour Perception \\
Test \\
5) Categorisation of \\
Synthesised Tonal Continua \\
6) Picture Naming \\
7) Questionnaire
\end{tabular}




\section{Figure 2}

Screen shot from the Training task.

The stimuli heard is ' $d i$ ', tone 4, [earth]. The foil picture on the right is 'di' tone 2, [siren].
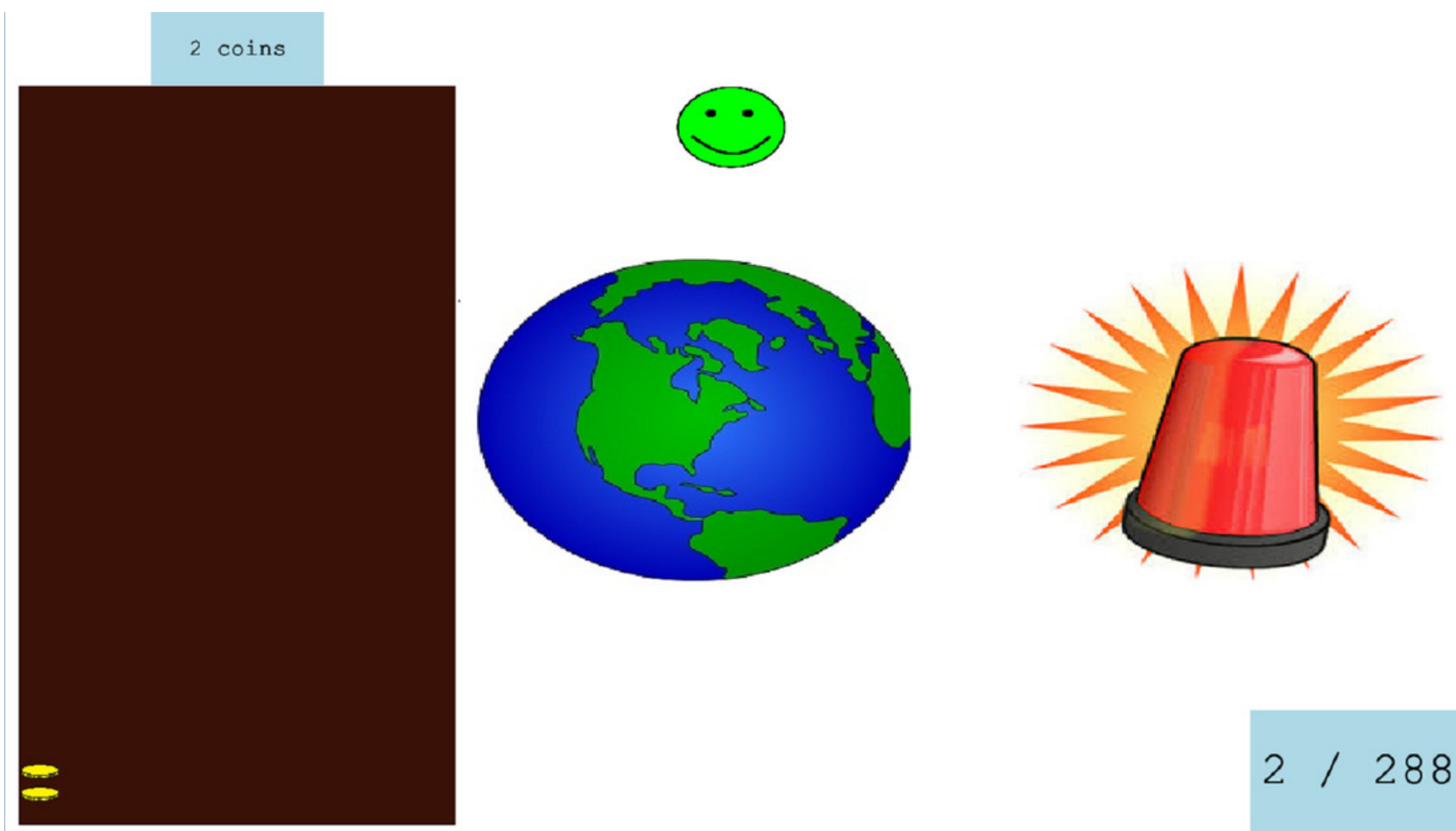
Figure 3

Mean accuracy for the LV (Low Variability), HV (High Variability) \& HVB (High Variability Blocked) groups in Pitch Contour Perception Test. Error bars represents the 95\% confidence intervals.

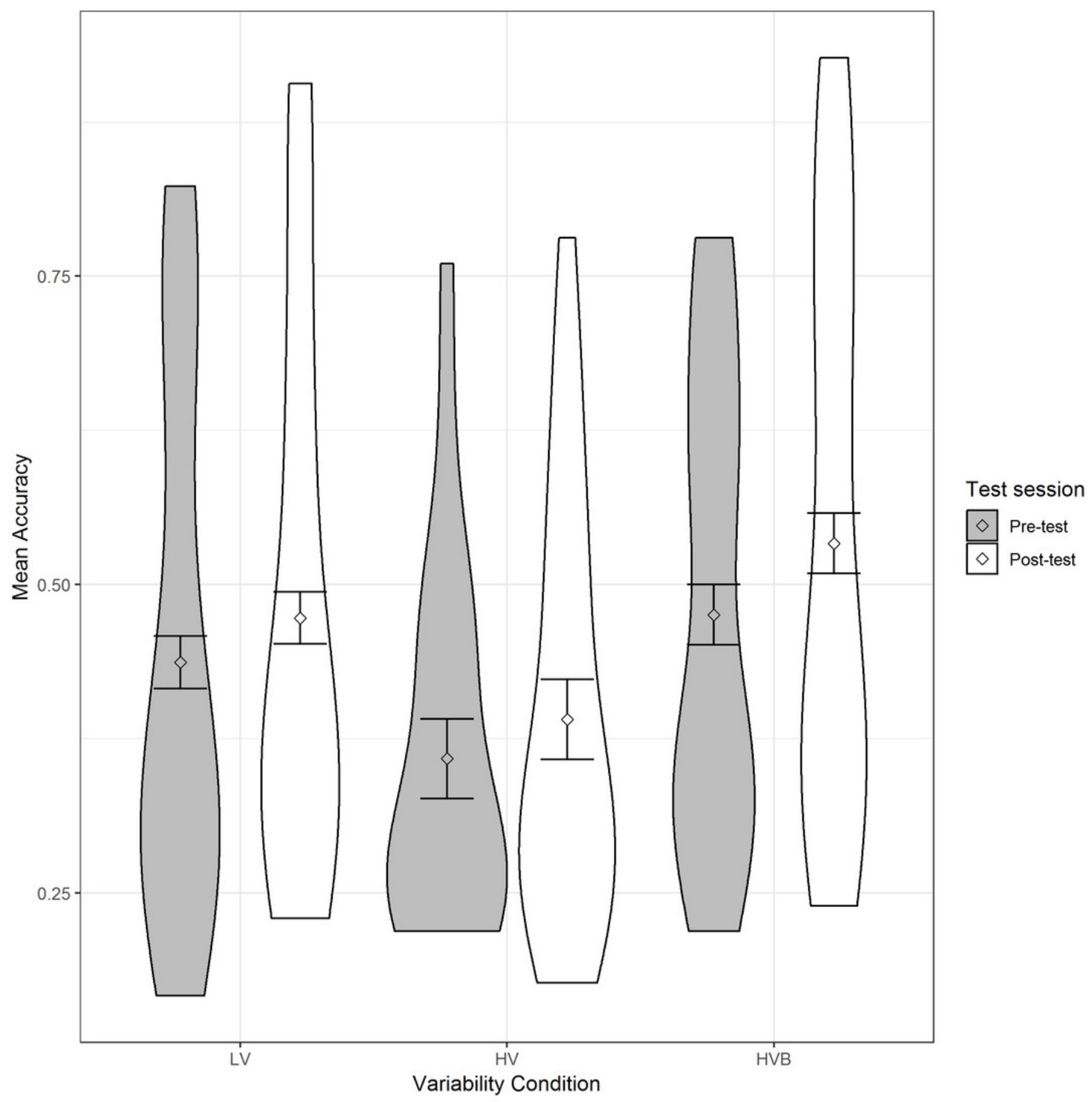


Figure 4

Mean accuracy in the Training task for the LV (Low Variability), HV (High Variability) and HVB (High Variability Blocked) training groups in each session. Y-axis starts from chance level. Error bars show 95\% confidence intervals.

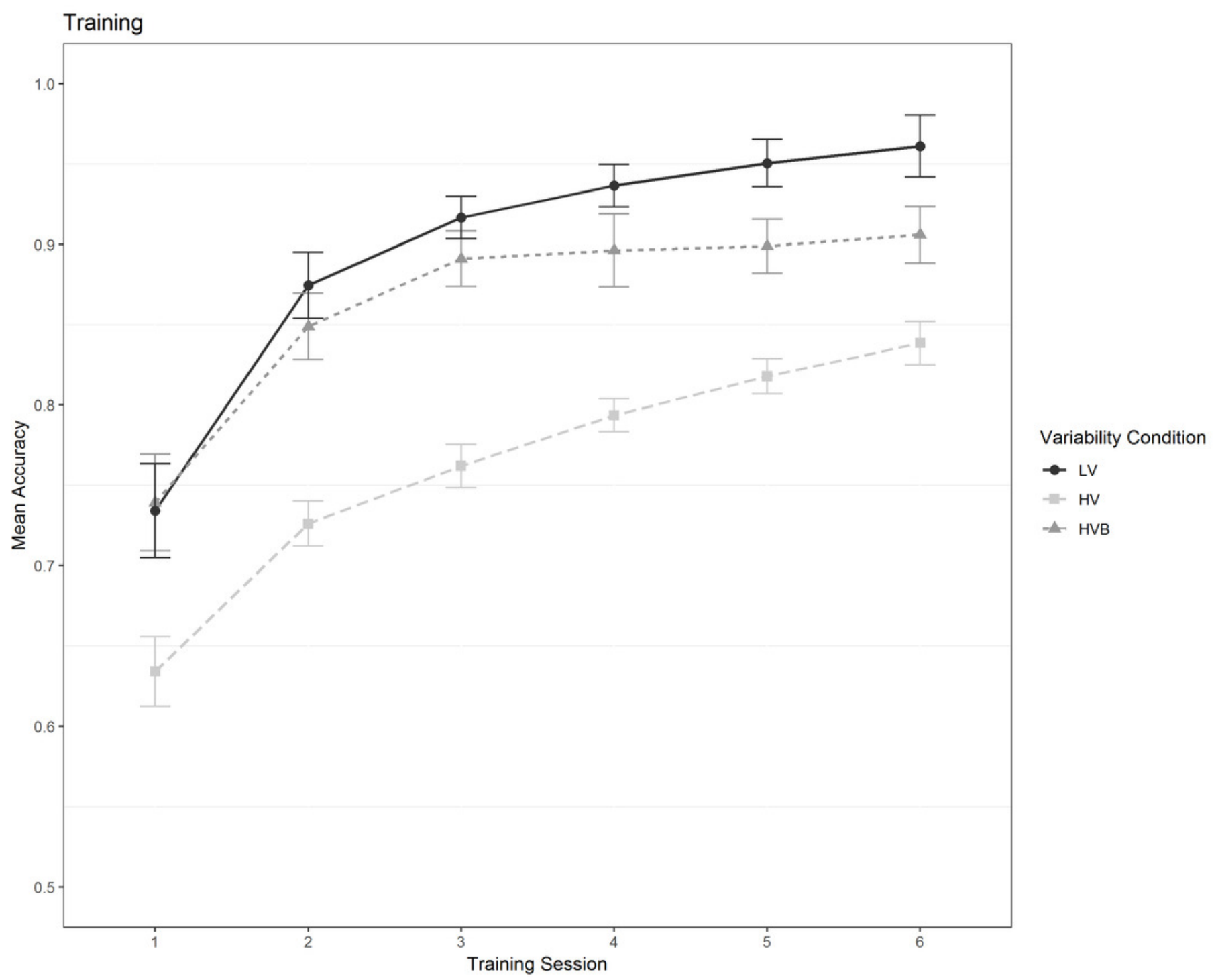


Figure 5

Mean accuracy in Three Interval Oddity task for LV (Low Variability), HV (High Variability) and HVB (High Variability Blocked) training groups in Pre- and Post-tests for trained and untrained items. Error bars show 95\% confidence intervals.

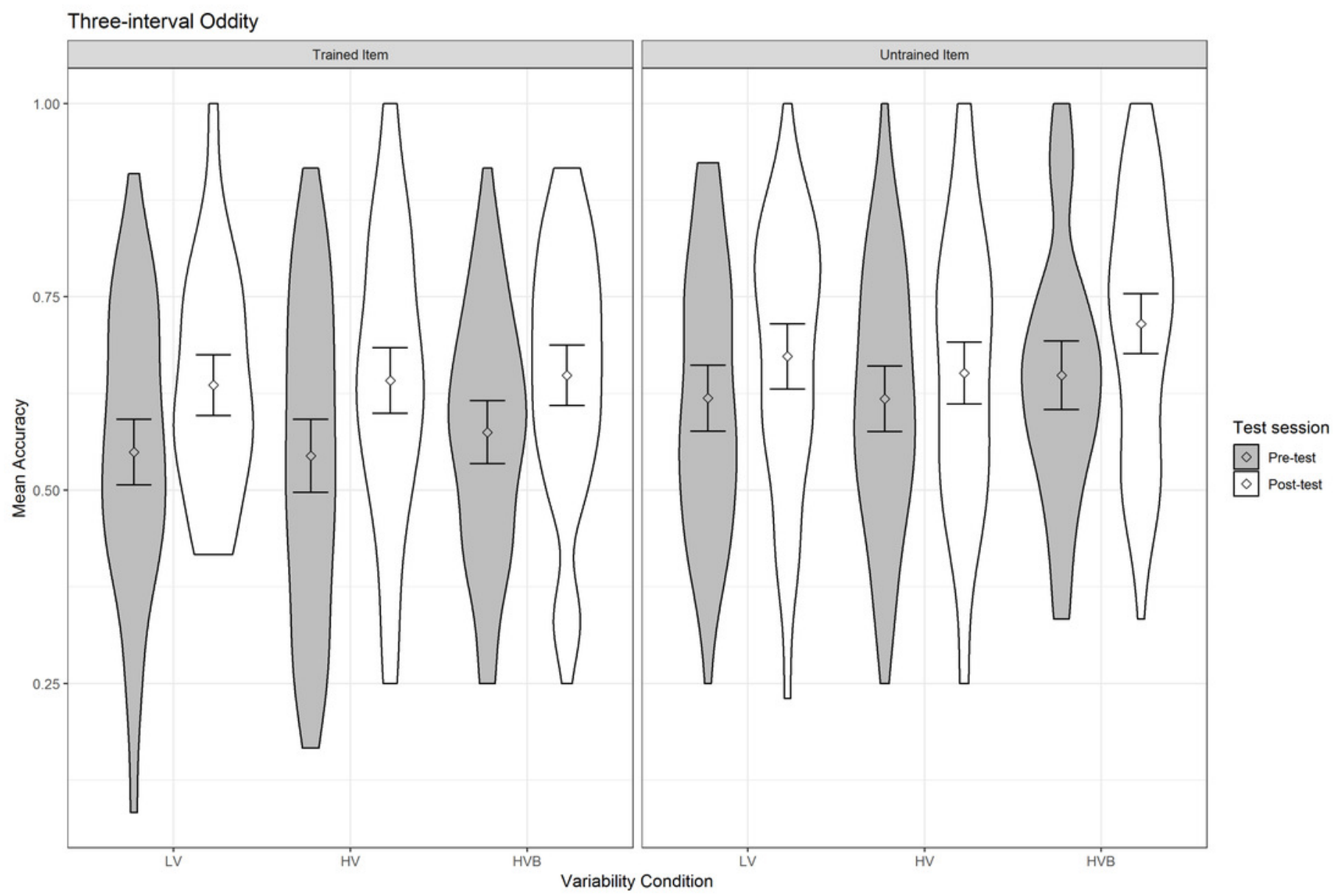


Figure 6

Mean accuracy of Picture Identification for LV (Low Variability), HV (High Variability) and HVB (High Variability Blocked) training groups for untrained voices and trained voices. Error bars show $95 \%$ confidence intervals.

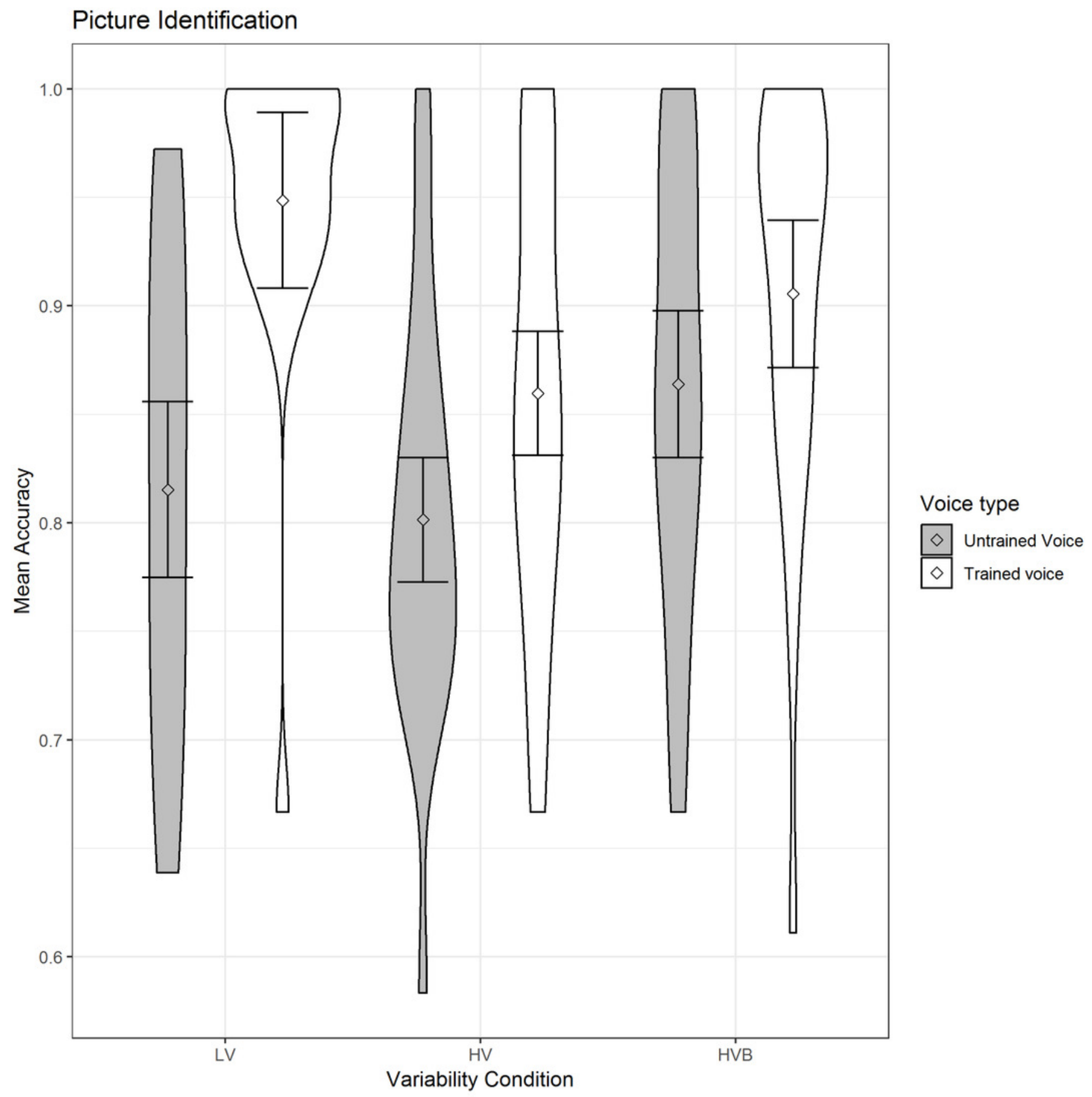


Figure 7

Accuracy of Word Repetition for LV (Low Variability), High Variability (HV) and High Variability Blocked (HVB) training groups in Pre- and Post-tests for trained and untrained items. Error bars show 95\% confidence intervals.

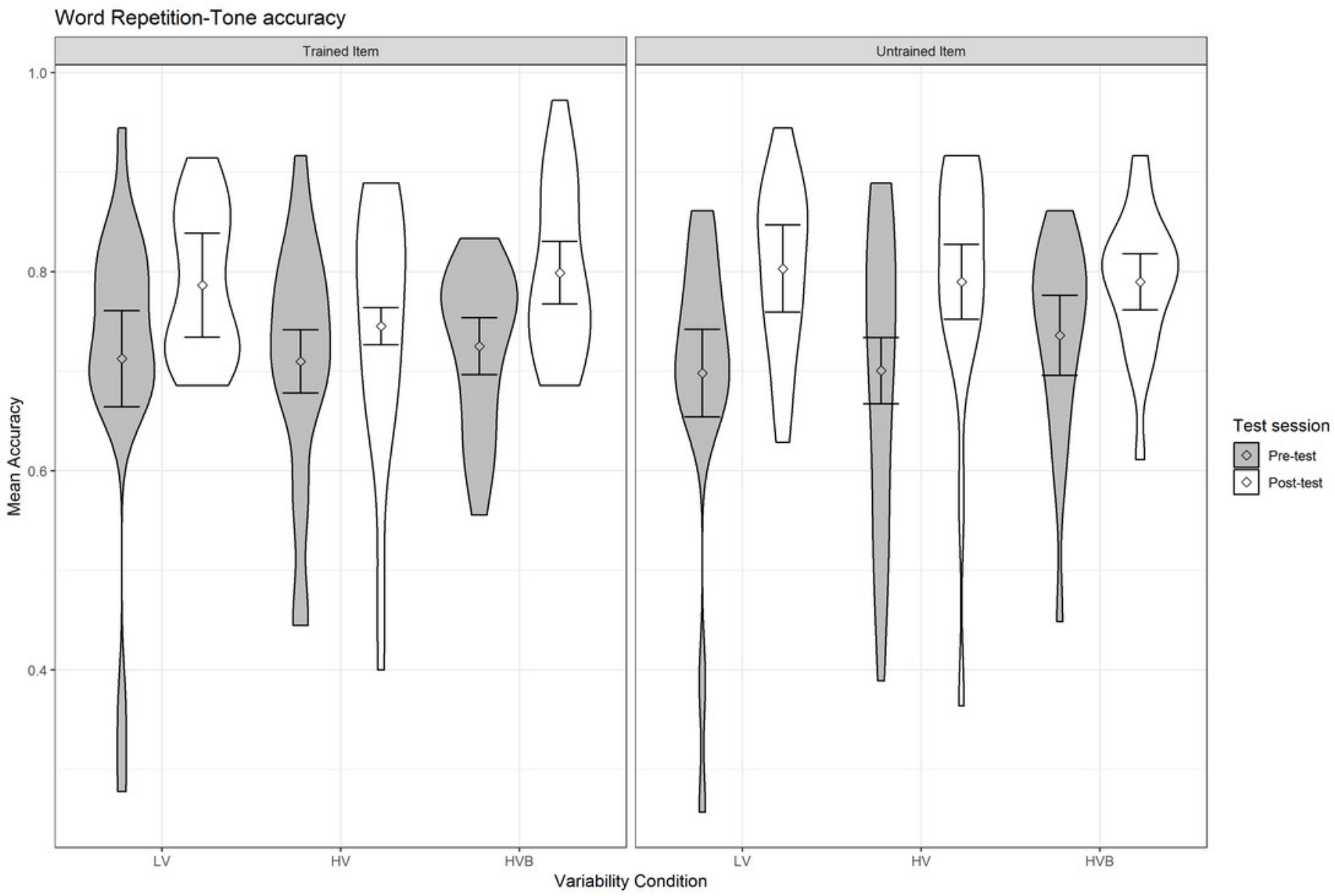




\section{Figure 8}

Mean pinyin accuracy of Word Repetition for LV (Low Variability), HV (High Variability) and HVB (High Variability Blocked) training groups in Pre- and Post-tests for trained and untrained items. Error bars show 95\% confidence intervals.

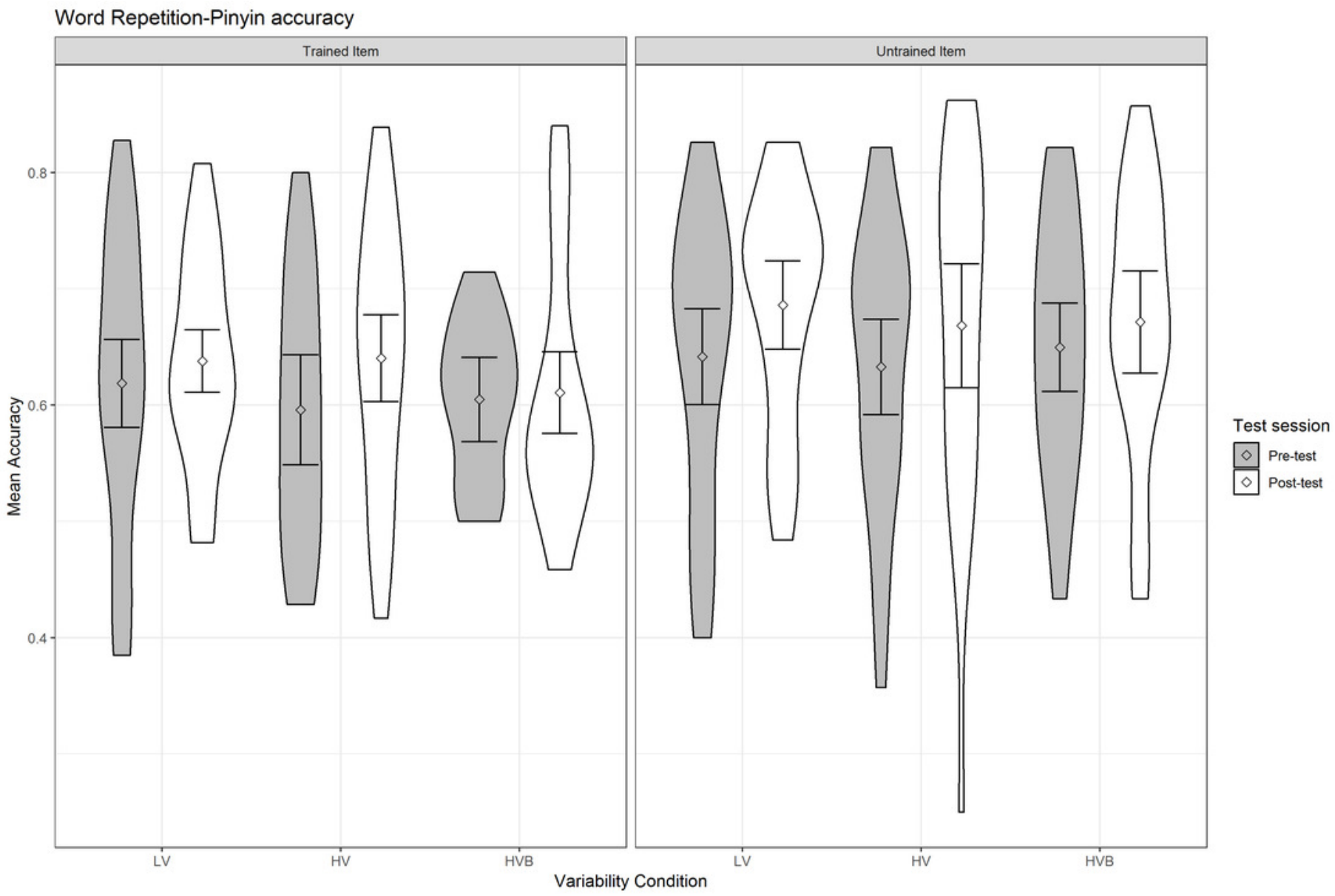


Figure 9

Tone accuracy and Pinyin accuracy of Picture Naming for LV (Low Variability), HV (High Variability) and HVB (High Variability Blocked) training groups. Error bars show 95\% confidence intervals.

A Picture Naming - Tone accuracy

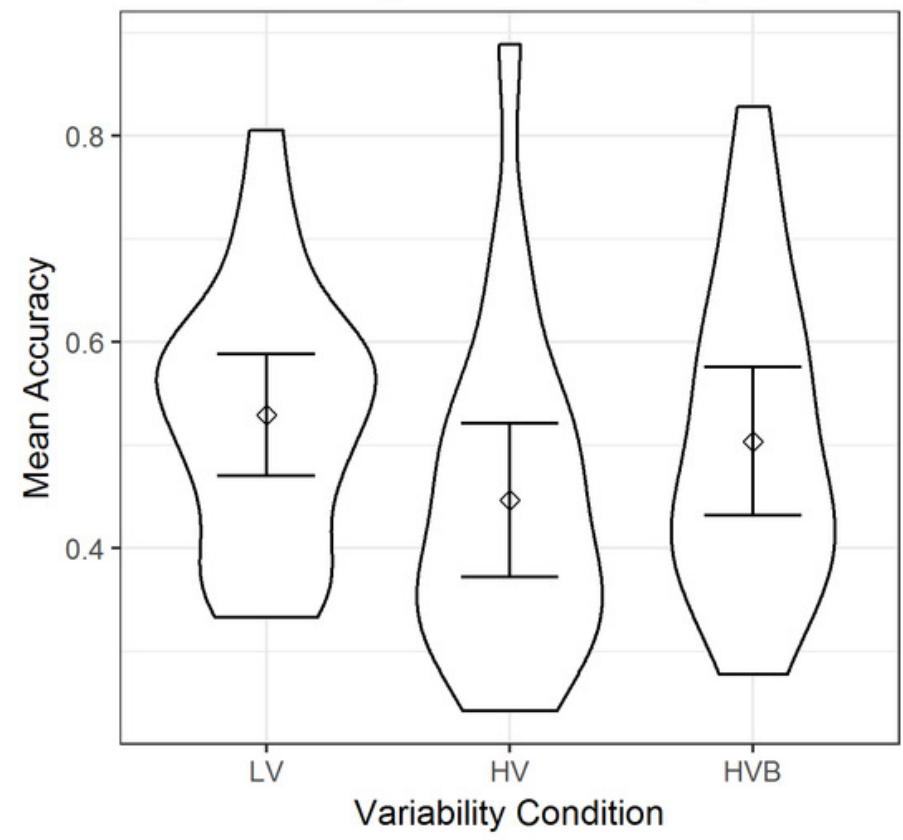

B Picture Naming - Pinyin Accuracy

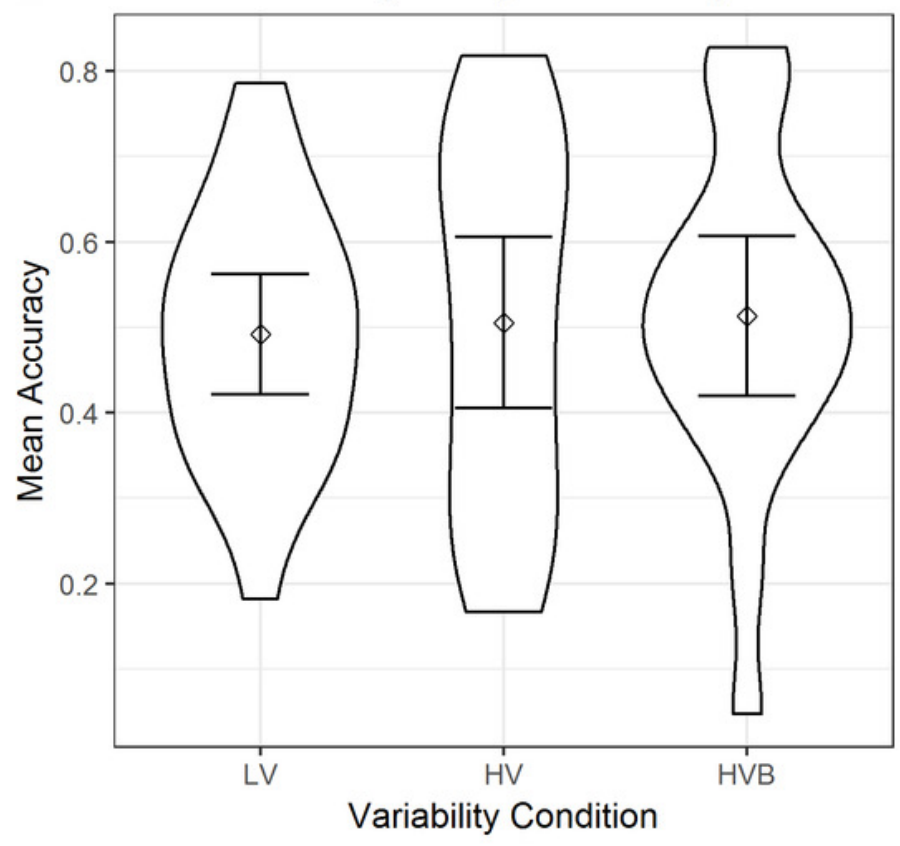




\section{Figure 10}

Accuracy in the Three Interval Oddity and Training data for LV (Low Variability), HV (High Variability) and HVB (High Variability Blocked) training groups. Error bars show $95 \%$ confidence interval.

(A)Mean accuracy of Three Interval Oddity, split by high (HA) versus low (LA) aptitude in the Pitch Contour Perception Test task (B) Mean accuracy of Training, split by high (HA) versus low (LA) aptitude in the Pitch Contour Perception Test task 
A Three-interval Oddity

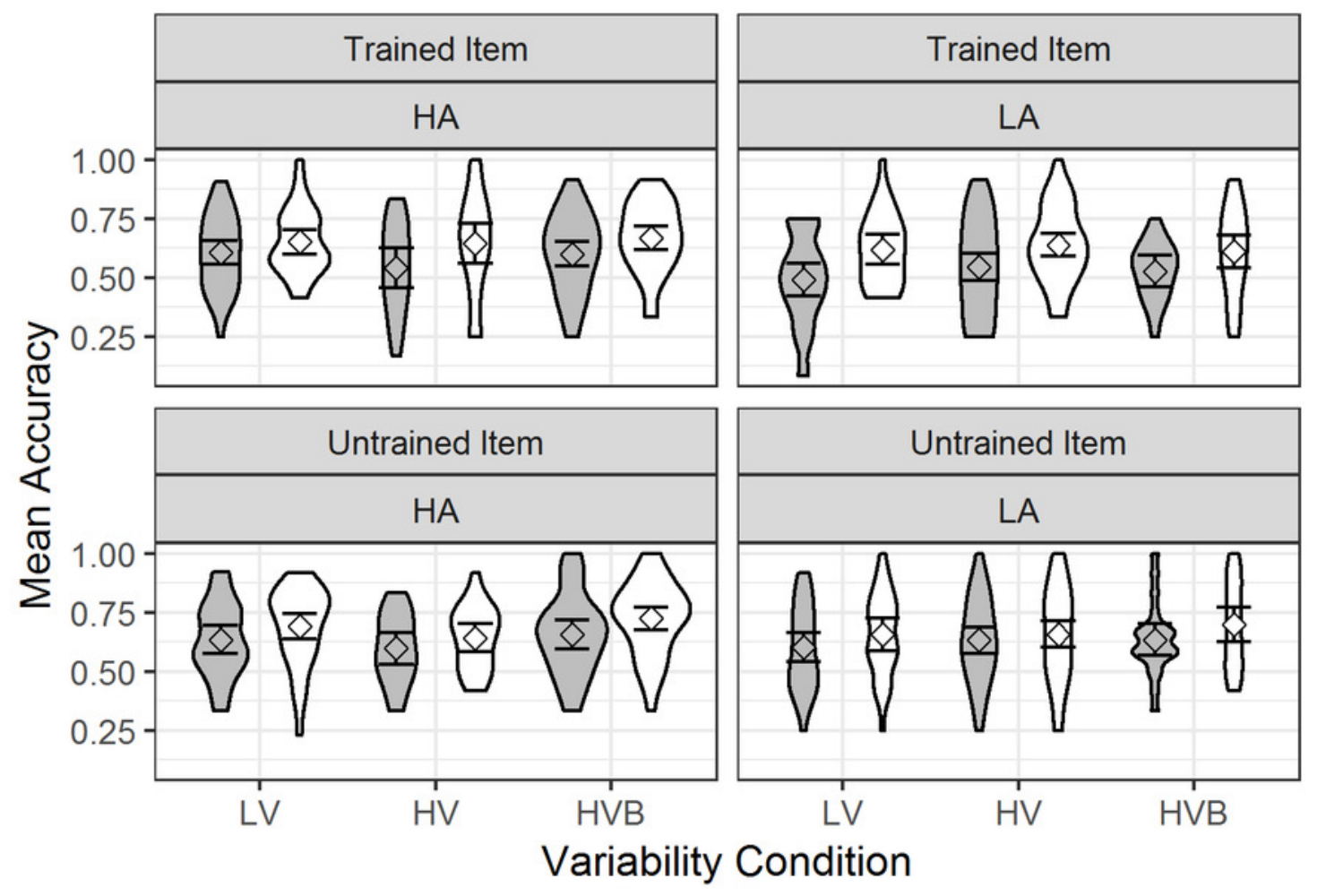

Test session \begin{tabular}{|l|l}
\hline$\diamond$ & Pre-test \\
\cline { 1 - 1 } & Post-test
\end{tabular}

B Training
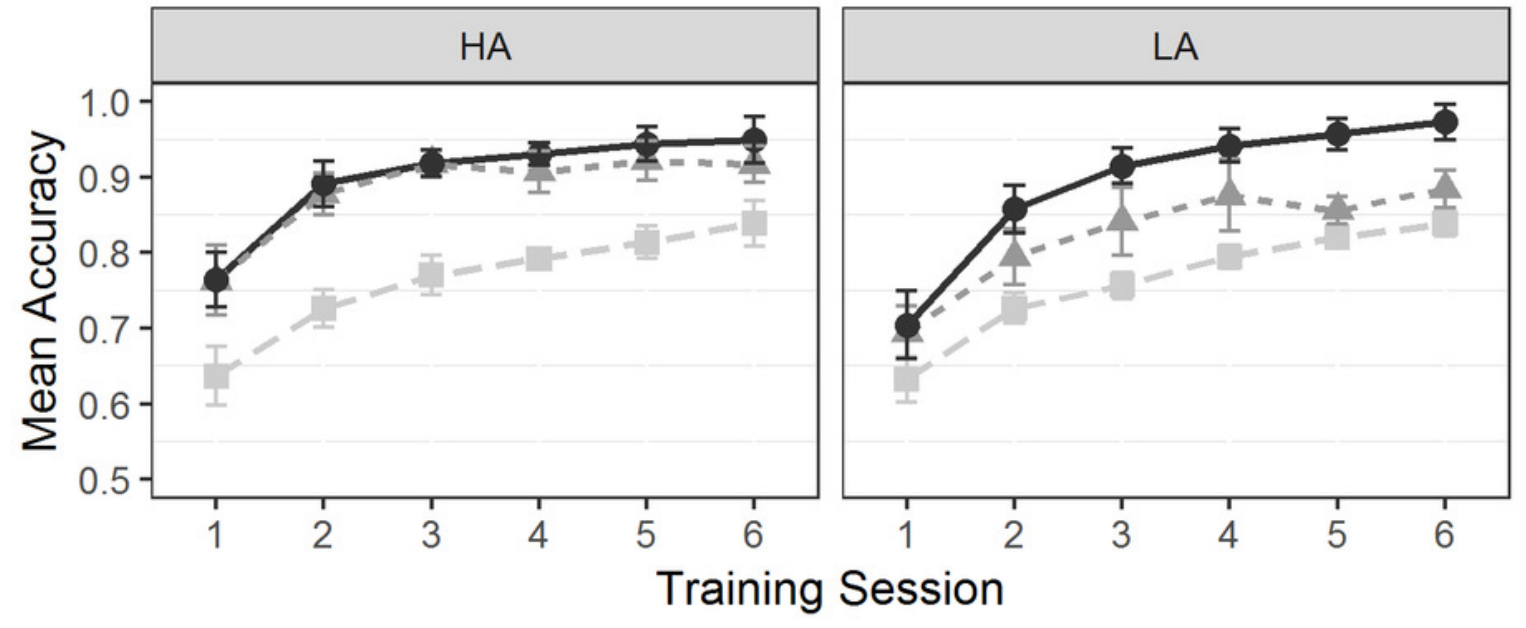

Condition

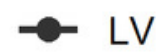

HV

$\therefore$ HVB 


\section{Figure 11}

Accuracy in the Picture Naming and Picture Identification data for LV , HV and HVB training groups, split by high (HA) versus low (LA) aptitude in the Pitch Contour Perception Test.

Error bars show 95\% confidence interval. (A) Mean accuracy of Picture Naming tone accuracy measure (B) Scatter plot contrasting Mean accuracy of Picture Naming tone accuracy measure and corresponding aptitude measure from Picture Contour Perception Test (C) Mean accuracy of Picture Naming Pinyin accuracy measure (D) Scatter plot contrasting Mean accuracy of Picture Naming Pinyin accuracy measure and corresponding aptitude measure from Picture Contour Perception Test (E) Mean accuracy of Picture Identification (F) Scatter plot contrasting Mean accuracy of Picture Identification and corresponding aptitude measure from Picture Contour Perception Test 
A Picture Naming - Tone accuracy

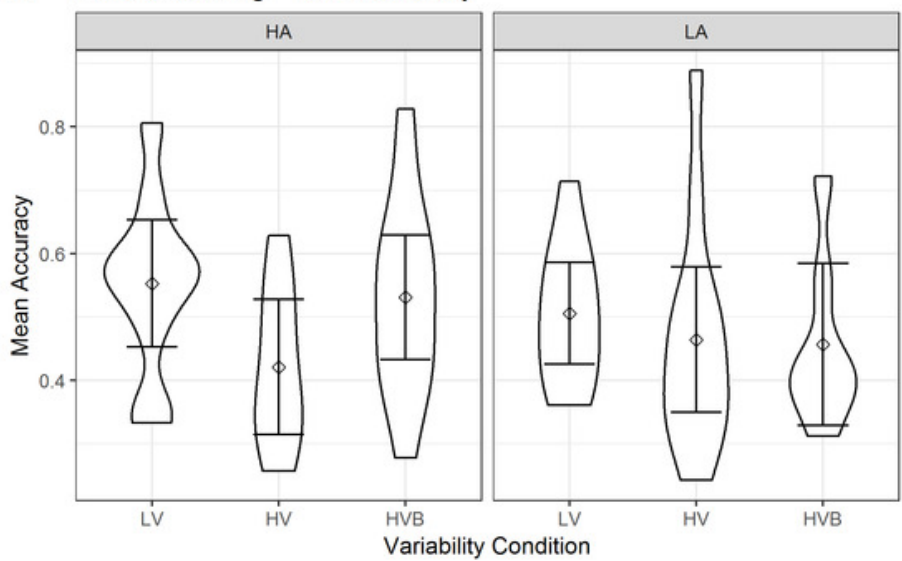

C Picture Naming - Pinyin Accuracy

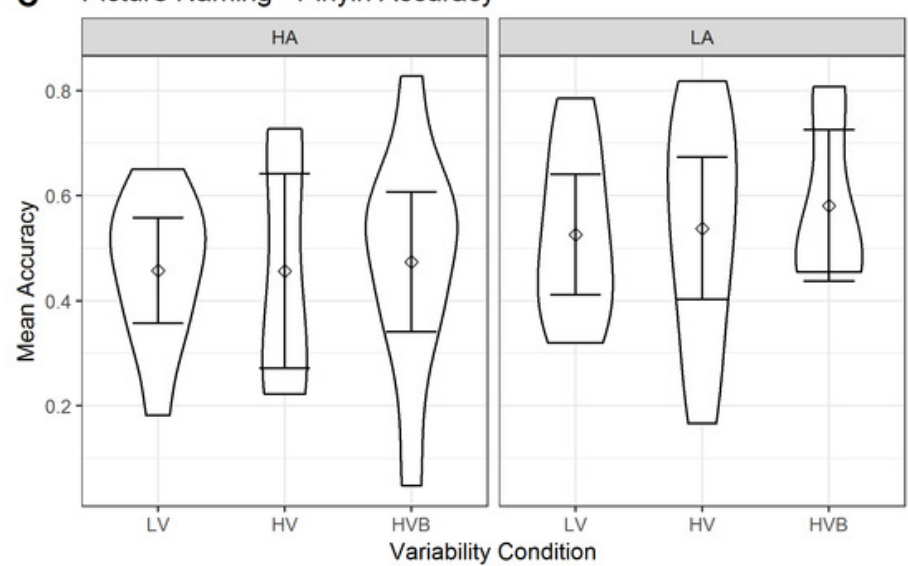

E Picture Identification

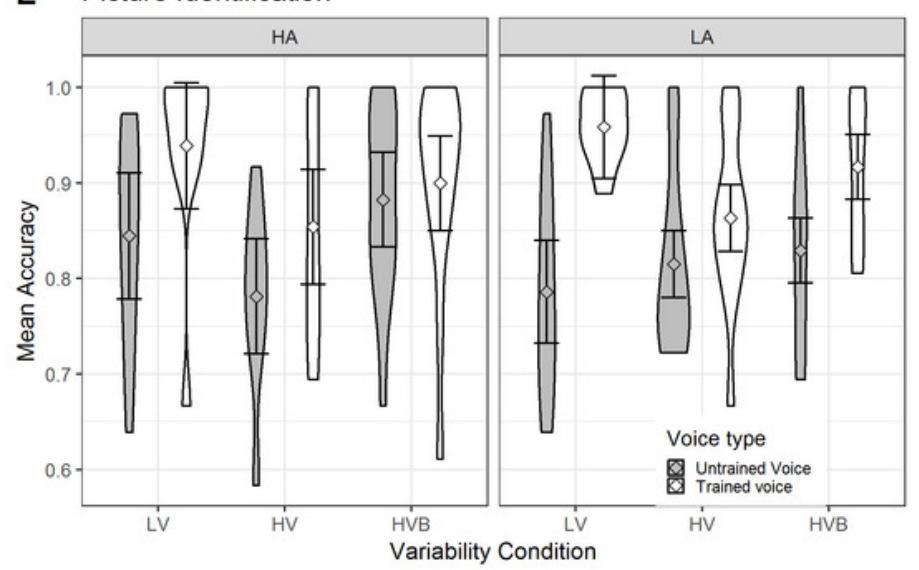

B

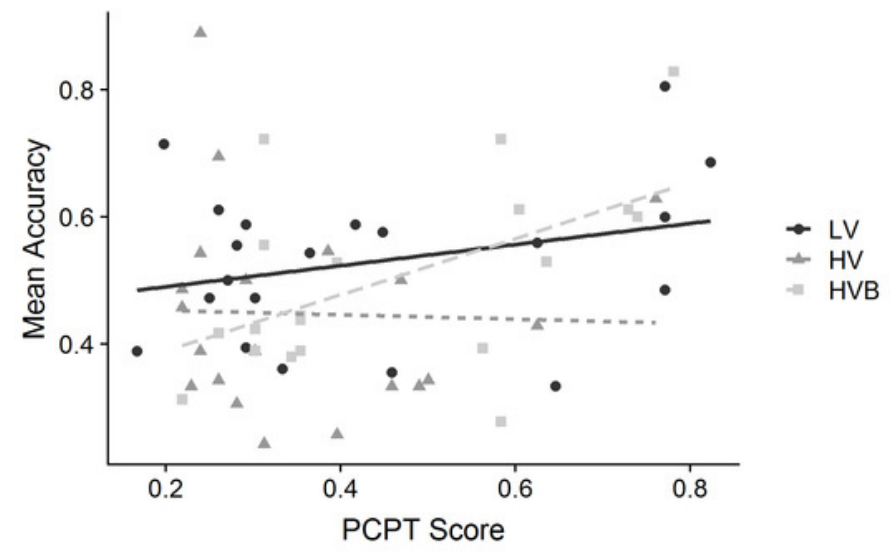

D

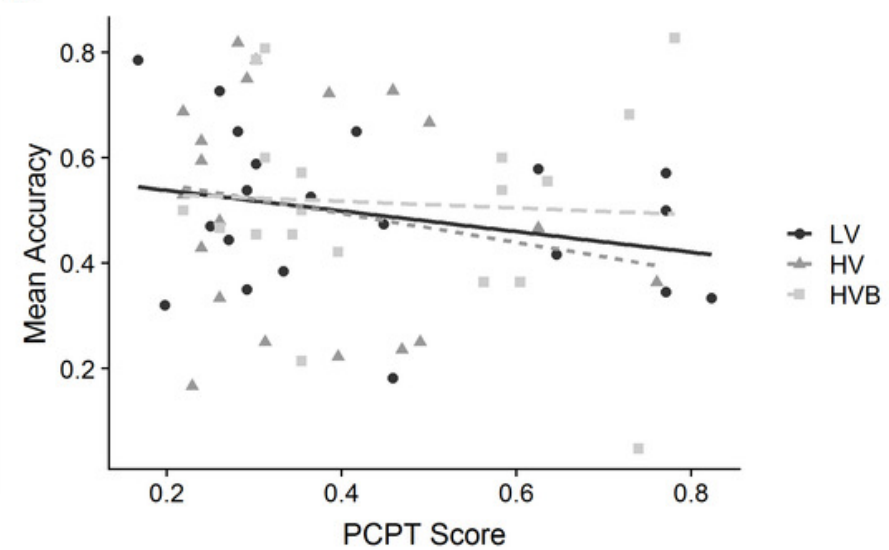

F

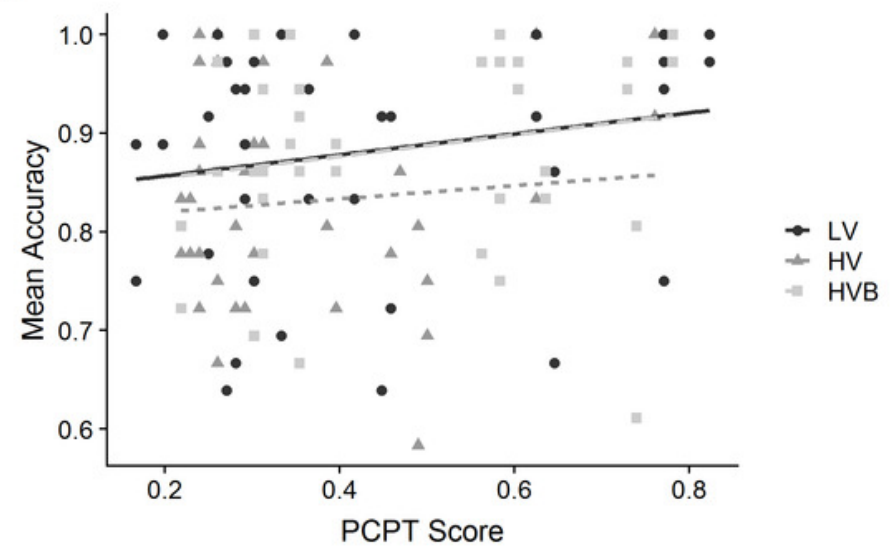


Figure 12

Accuracy in the Word Repetition data for LV, HV and HVB training groups, split by high (HA) versus low (LA) aptitude in the Pitch Contour Perception Test. Error bars show 95\% confidence intervals.

(A) Mean accuracy of Word Repetition tone accuracy measure (B) Mean accuracy of Word Repetition Pinyin accuracy measure

A Word Repetition-Tone accuracy

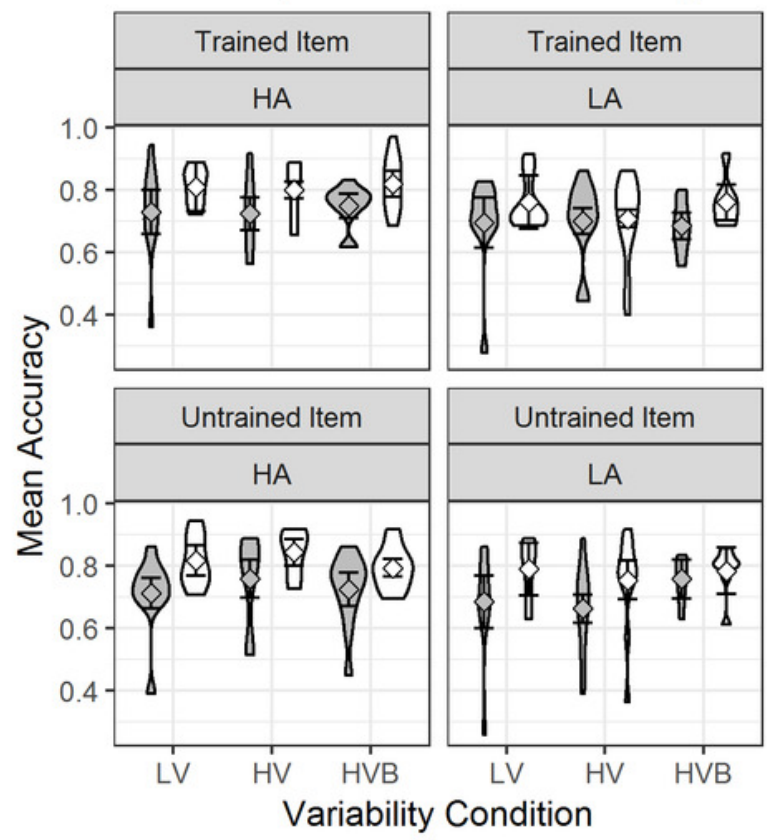

B Word Repetition-Pinyin accuracy

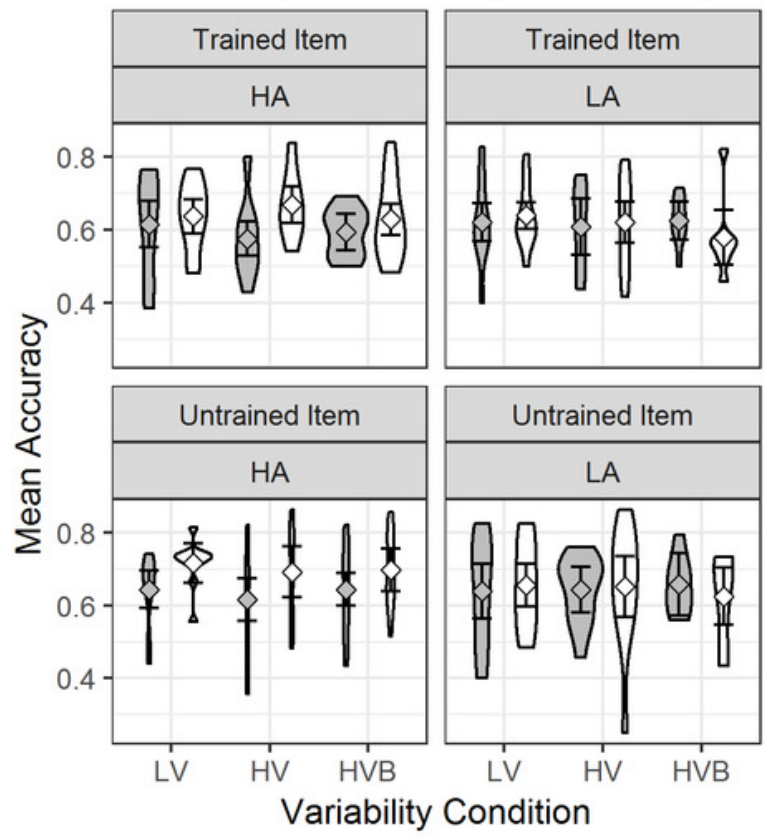

Test session

$\diamond$ Pre-test

$\diamond$ Post-test 


\section{Table $\mathbf{1}$ (on next page)}

Mean age range, average number of languages learned and mean starting age of learning the first L2 for participants in each condition. 
1

\begin{tabular}{lcccc}
\hline Condition & Mean Age & Age Range & Languages & Average Staring \\
& & & Learned & Age \\
\hline Low Variability & $26.15(2.2)$ & $19-53$ & $2.7(0.5)$ & $13.8(1.1)$ \\
High Variability & $25.65(0.7)$ & $19-47$ & $2.5(0.6)$ & $12.2(0.5)$ \\
High Variability & $22.05(1.4)$ & $19-30$ & $2.0(1.3)$ & $11.8(0.4)$ \\
blocked & & & & \\
\hline
\end{tabular}

2 
Table 2 (on next page)

Use of trained and untrained items and voices in different tasks. 


\begin{tabular}{ccc}
\hline Task & Items & Voice \\
\hline Picture Identification & Trained & $\begin{array}{c}\text { One trained voice } \\
\text { (counterbalanced, see Table 3) } \\
\text { One untrained voice }\end{array}$ \\
(counterbalanced, see Table 3)
\end{tabular}

2 


\section{Table 3(on next page)}

Counterbalancing of voices across training conditions in the Picture Identification task (the only test in which trained and untrained voices are directly contrasted) and the Word Repetition tests. 


\begin{tabular}{cccccc}
\hline Task & & & Voice & & \\
& Version 1 & Version 2 & Version 3 & Version 4 & Version 5 \\
\hline Training, LV & F1 & F2 & F3 & M1 & M2 \\
\hline Training, HV/HVB & F1 & F2 & F3 & M1 & M2 \\
& F3 & F1 & M2 & F1 & F2 \\
& M1 & M1 & F1 & F2 & F3 \\
& M2 & M2 & F2 & F3 & M1 \\
\hline Picture Identification & & & & & \\
Trained voice & F1 & F2 & F3 & M1 & M2 \\
Untrained voice & F2 & F3 & M1 & M2 & F1 \\
\hline Word Repetition & F1 & F2 & F3 & M1 & M2
\end{tabular}

2 
Table 4 (on next page)

Statistics obtained when adding in participant aptitude (as measured by performance on the Pitch Contour Perception Test task at pre-test) into the models predicting performance on the test and training tasks. 


\begin{tabular}{|c|c|c|}
\hline Data Set & Coefficient Name & Statistics \\
\hline Word Repetition: & Aptitude & $\beta=0.07, S E=0.03, z=2.35, p=.019$ \\
\hline Tone Accuracy & Aptitude by Test-Session & $\beta=0.03, S E=0.04, z=0.72, p=.473$ \\
\hline \multirow[t]{6}{*}{ (Pre/Post) } & Aptitude by LV-HV Contrast by Test-Session & $\beta=0.05, S E=0.11, z=0.47, p=.639$ \\
\hline & Aptitude by LV-HVB Contrast by Test-Session & $\beta=0.13, S E=0.10, z=1.35, p=.176$ \\
\hline & Aptitude by LV-HV Contrast by Test-Session by & $\beta=-0.14, S E=0.15, z=-0.97, p=.334$ \\
\hline & Item-Novelty & \\
\hline & Aptitude by LV-HVB Contrast by Test-Session by & $\beta=0.07, S E=0.13, z=0.50, p=.61$ \\
\hline & Item-Novelty & \\
\hline Three Interval & Aptitude & $\beta=0.07, \mathrm{SE}=0.03, z=2.19, p=.029$ \\
\hline Oddity & Aptitude by Test-Session & $\beta=0.01, S E=0.23, z=0.31, p=.757$ \\
\hline \multirow[t]{6}{*}{ (Pre/Post) } & Aptitude by LV-HV Contrast by Test-Session & $\beta=0.05, S E=0.07, z=0.77, p=.443$ \\
\hline & Aptitude by LV-HVB Contrast by Test-Session & $\beta=0.05, S E=0.06, z=0.83, p=.410$ \\
\hline & Aptitude by LV-HV Contrast by Test-Session by & $\beta=-0.12, S E=0.13, z=-0.94, p=.346$ \\
\hline & Item-Novelty & \\
\hline & Aptitude by LV-HVB Contrast by Test-Session by & $\beta=0.06, S E=0.11, z=0.52, p=.604$ \\
\hline & Item-Novelty & \\
\hline \multirow[t]{2}{*}{ Training } & Aptitude & $\beta=0.13, S E=0.048, z=2.70, p=.007$ \\
\hline & Aptitude by LV-HV Contrast & $\beta=-0.04, S E=0.11, z=-0.332, p=.740$ \\
\hline
\end{tabular}

Aptitude by LV-HV Contrast

$\beta=0.03, \mathrm{SE}=0.10, z=0.26, p=0.795$ 


\begin{tabular}{|c|c|c|}
\hline \multirow[t]{4}{*}{ (Post Only) } & Aptitude by LV-HV Contrast & $\beta=-0.02, S E=0.19, z=-0.12, p=.901$ \\
\hline & Aptitude by LV-HVB Contrast & $\beta=0.01, S E=0.17, z=0.09, p=.932$ \\
\hline & Aptitude by LV-HV Contrast by Voice-Novelty & $\beta=0.35, S E=0.21, z=1.63, p=.103$ \\
\hline & Aptitude by LV-HVB Contrast by Voice-Novelty & $\beta=-0.11, S E=0.19, z=-0.58, p=.566$ \\
\hline Picture Naming: & Aptitude & $\beta=0.08, \mathrm{SE}=0.04, z=1.89, p=.0 .059$ \\
\hline \multirow[t]{2}{*}{ Tone Accuracy } & Aptitude by LV-HV Contrast & $\beta=-0.09, \mathrm{SE}=0.11, z=-0.84, p=.402$ \\
\hline & Aptitude by LV-HVB Contrast & $\beta=0.12, S E=0.10, z=1.22, p=.224$ \\
\hline
\end{tabular}

2 


\section{Table 5 (on next page)}

Bayes Factor results testing the hypothesis that there is greater generalisation following either of the high variability training conditions than the low variability condition 


\begin{tabular}{cccccc}
\hline Contrast & $\begin{array}{c}\text { Mean } \\
\text { difference }\end{array}$ & $\begin{array}{c}\text { Stand. } \\
\text { Error }\end{array}$ & $\begin{array}{c}\text { H1 } \\
\text { estimate } \boldsymbol{x}\end{array}$ & $\begin{array}{c}\text { Bayes } \\
\text { Factor (B) }\end{array}$ & $\begin{array}{c}\text { Robustness } \\
\text { Region }\end{array}$ \\
\cline { 2 - 5 } $\begin{array}{c}\text { Picture ID (Novel voice only) } \\
\text { HV+ HVB }>\text { LV }\end{array}$ & 0.13 & 0.228 & 1.71 & 0.219 & $1.11: \infty$ \\
$\begin{array}{c}\text { Picture Naming, (Tone accuracy) } \\
\text { HV+ HVB }>\text { LV }\end{array}$ & -0.225 & 0.168 & 1.076 & 0.067 & $0.202: \infty$ \\
$\begin{array}{c}\text { Picture Naming (Pinyin Accuracy) } \\
\text { HV+ HVB }>\text { LV }\end{array}$ & 0.104 & 0.196 & 4.05 & 0.08 & $0.101: \infty$ \\
$\begin{array}{c}\text { Word Repetition (Tone accuracy) } \\
\text { test-session by } \\
H V+H V B>L V\end{array}$ & -0.108 & 0.157 & 0.395 & 0.239 & \\
$\begin{array}{c}\text { Word Repetition (Pinyin accuracy) } \\
\text { test-session by HV+ HVB }>\text { LV }\end{array}$ & 0.095 & -0.034 & 0.152 & $0.303: \infty$ \\
$\begin{array}{c}\text { Three Interval Oddity } \\
\text { test-session by } H V+H V B>L V\end{array}$ & -0.001 & 0.1 & 0.31 & 0.303 & 0.202 \\
\hline
\end{tabular}




\section{Table 6(on next page)}

Bayes Factor results testing the hypothesis that there is an interaction between aptitude and variability-condition greater generalisation following either of the high variability training conditions than the low variability condition 


\begin{tabular}{|c|c|c|c|c|c|}
\hline Contrast & $\begin{array}{c}\text { Mean } \\
\text { difference }\end{array}$ & Stand. Error & H1 estimate $x$ & $\begin{array}{l}\text { Bayes } \\
\text { Factor }(B)\end{array}$ & $\begin{array}{l}\text { Robustness } \\
\text { Region }\end{array}$ \\
\hline $\begin{array}{c}\text { ID, (Tone accuracy) } \\
\text { aptitude by HV+ HVB > LV }\end{array}$ & 0.006 & 0.127 & 0.171 & 0.617 & $0: 0.354$ \\
\hline $\begin{array}{c}\text { Picture Naming, (Tone accuracy) } \\
\text { aptitude by HV+ HVB }>\text { LV }\end{array}$ & 0.042 & 0.083 & 0.099 & 0.904 & $0: 0.354$ \\
\hline $\begin{array}{l}\text { Three Interval Oddity (Tone accuracy) } \\
\text { aptitude by test-session by HV+ HVB }>\mathrm{LV}\end{array}$ & 0.048 & 0.05 & 0.345 & 0.371 & $0: 0.354$ \\
\hline $\begin{array}{l}\text { Word Repetition (Tone accuracy) } \\
\text { aptitude by test-session by HV+ HVB }>\mathrm{LV}\end{array}$ & 0.091 & 0.082 & 0.379 & 0.654 & $0: 0.758$ \\
\hline $\begin{array}{c}\text { Training } \\
\text { aptitude by } \mathrm{HV}>\mathrm{LV}\end{array}$ & -0.037 & 0.119 & 0.129 & 0.572 & $0: 0.253$ \\
\hline $\begin{array}{l}\text { Training } \\
\text { aptitude by HVB }>\mathrm{LV}\end{array}$ & 0.026 & 0.101 & 0.129 & 0.732 & $0: 0.354$ \\
\hline
\end{tabular}

